\title{
The extended Görtler-Hämmerlin model for linear instability of three-dimensional incompressible swept attachment-line boundary layer flow
}

\author{
By VASSILIOS THEOFILIS ${ }^{1}$, ALEXANDER FEDOROV ${ }^{\dagger}$, \\ DOMINIK OBRIST $T^{3}$ AND UWE Ch. DALLMAN ${ }^{1 \dagger}$ \\ ${ }^{1}$ DLR Institute of Fluid Mechanics, Transition and Turbulence, \\ Bunsenstraße 10, D-37073 Göttingen, Germany \\ ${ }^{2}$ Moscow Institute of Physics and Technology, 141700 Moscow Region, Russia \\ ${ }^{3}$ Cray Computer GmbH, Zielstattstraße 10a, D-81379 München, Germany
}

A simple extension of the classic Görtler-Hämmerlin (1955) (GH) model, essential for three-dimensional linear instability analysis, is presented. The extended GörtlerHämmerlin model classifies all three-dimensional disturbances in this flow by means of symmetric and antisymmetric polynomials of the chordwise coordinate. It results in one-dimensional linear eigenvalue problems, a temporal or spatial solution of which, presented herein, is demonstrated to recover results otherwise only accessible to the temporal or spatial partial-derivative eigenvalue problem (the former also solved here) or to spatial direct numerical simulation (DNS). From a numerical point of view, the significance of the extended GH model is that it delivers the three-dimensional linear instability characteristics of this flow, discovered by solution of the partial-derivative eigenvalue problem by Lin \& Malik (1996a), at a negligible fraction of the computing effort required by either of the aforementioned alternative numerical methodologies. More significant, however, is the physical insight which the model offers into the stability of this technologically interesting flow. On the one hand, the dependence of three-dimensional linear disturbances on the chordwise spatial direction is unravelled analytically. On the other hand, numerical results obtained demonstrate that all linear three-dimensional instability modes possess the same (scaled) dependence on the wallnormal coordinate, that of the well-known $\mathrm{GH}$ mode. The latter result may explain why the three-dimensional linear modes have not been detected in past experiments; criteria for experimental identification of three-dimensional disturbances are discussed. Asymptotic analysis based on a multiple-scales method confirms the results of the extended GH model and provides an alternative algorithm for the recovery of three-dimensional linear instability characteristics, also based on solution of onedimensional eigenvalue problems. Finally, the polynomial structure of individual three-dimensional extended GH eigenmodes is demonstrated using three-dimensional DNS, performed here under linear conditions.

$\dagger$ Present address: Rockwell Scientific, P.O. Box 1085, Thousand Oaks, CA 91358, USA.

$\uparrow$ Dr Dallmann died during the late stages of revision of this work. 


\section{Introduction}

Steady laminar flow in the stagnation region of a swept cylindrical body is typically modelled by the swept Hiemenz boundary layer (Rosenhead 1963). The mathematically attractive feature of this flow, unlike that in the classic flat-plate boundary layer, is that it represents an exact solution of the incompressible continuity and Navier-Stokes equations. The limitations of this flow model are encountered when compressibility or curvature are introduced. Within its realm of applicability, justification for the use of the swept Hiemenz basic flow model in performing linear and nonlinear stability analyses is provided by the agreement of this model with the experimentally obtained steady laminar basic flow (Gaster 1967) and the consequent agreement of the linear stability results pertinent to the swept Hiemenz flow with those obtained in a series of experiments performed under conditions favouring the growth of small-amplitude disturbances (Pfenninger \& Bacon 1969; Poll 1979; Hall, Malik \& Poll 1984; Arnal, Coustols \& Juillen 1984; Poll, Danks \& Yardley 1996). The stability characteristics of the swept Hiemenz boundary layer flow have also been recovered in the three-dimensional direct numerical simulation (DNS) of Spalart (1988). One of the most significant results of the latter work has been the demonstration that the most unstable (least stable) eigenmode emerging out of whitenoise initial disturbances is one which satisfies an assumption proposed by Görtler (1955) and Hämmerlin (1955) (hereafter referred to as GH) according to which linear instabilities in the attachment-line boundary layer inherit the symmetry of the basic (unswept) Hiemenz flow, their chordwise velocity component being a linear function of the chordwise coordinate $x$, while the wall-normal velocity component is independent of $x$. This assumption has been extended by Hall et al. (1984) in the swept Hiemenz flow, in which the GH Ansatz for the linear perturbations is retained and extended to assume a spanwise disturbance velocity component which is independent of the chordwise coordinate. Lack of capacity to solve the appropriate two-dimensional eigenvalue problem numerically resulted in the GH Ansatz being considered, until the simulation of Spalart, as an assumption of mathematical convenience, as its authors also presented it.

While Dallmann (1980) recast the stability problem of flow in the stagnation region of a swept cylindrical body into a system of separable equations in which the GH structure is only one of those permissible, it was Hall et al. (1984) who obtained the first numerical solutions to the temporal one-dimensional eigenvalue problem resulting from linearization of the incompressible continuity and NavierStokes equations upon substitution of the GH structure into the equations of motion and obtained a $R e_{\text {crit }} \approx 583.1$; the corresponding spatial eigenvalue problem was solved numerically by Theofilis (1995). The eigenvalue problem results turned out to compare very well with experiment and two-dimensional DNS performed under conditions favouring linear growth (Hall \& Malik 1986; Jiménez et al. 1990; Theofilis $1998 b)$. In the latter work a close comparison with more recent experimental results (Poll et al. 1996) is also presented, which shows that the peak of the growing eigenmode corresponds in terms of frequency to the GH mode. However the error bar in the only experimentally available information besides neutral points, namely the frequency of the linear disturbances, appeared to be large. An explanation of the latter observation may be obtained by reference to the two-dimensional linear stability eigenvalue problem solutions presented by Lin \& Malik (1996a). The novelty in the work of these authors is that they resolved a direction additional to the wallnormal, namely that along the chord. Further, by analogy to plane Poiseuille flow, 
Lin \& Malik (1996a) imposed boundary conditions of symmetry or antisymmetry across the attachment line and discovered a sequence of eigenmodes, additionally to the well-understood $\mathrm{GH}$ perturbation, all of which are less amplified (stronger damped) than the GH mode, while their frequencies lie very close to that of the latter disturbance. Lin \& Malik (1996a) presented results without providing an explanation as to why additional modes should exist; Joslin (1996b) on the other hand, in his spatial DNS study of the spatial-theory analogues of the modes discovered by Lin \& Malik (1996a), mentions that asymptotic analysis revealed that such a sequence of three-dimensional instability modes is present in the flow at hand; this analysis is incorporated in the present work.

To date, there exist two most significant unresolved issues in the stability of the swept attachment-line boundary layer, both of which appear to be beyond the reach of one-dimensional linear or nonlinear analyses based on the classic GH structure. Firstly, this flow is subcritically unstable, and secondly the relation of instability at the attachment line to that downstream in the chordwise direction is not well understood. The first problem is related to the existence of a critical Reynolds number, $R e \approx 245$, above which turbulent flow has been documented both in the experiments of Poll (1979) and the DNS of Spalart (1988); this Reynolds number value appears to be unrelated to that which linear analyses deliver, $R e \approx 583$. The issue to be clarified in the second problem is the mechanism which feeds the region downstream in the chordwise direction with instability originating at the attachment line, and the precise relation of this mechanism to crossflow instability.

Evidence exists that both problems are three-dimensional and nonlinear in nature. With respect to the second question, Spalart (1989) has performed DNS in which he obtained solutions off the attachment line which are strongly reminiscent of crossflow vortices. Although crossflow vortices are related with the inviscidly unstable inflectional profile of the basic state, the fact that Spalart used the swept Hiemenz flow as a basic flow model suggests that the three-dimensional attachment-line boundary layer has the potential to generate crossflow-like instability outside the stagnation region. More recently, Bertolotti (2000) revisited this issue and showed that a class of eigenmodes connected with crossflow instability exists and is distinct from that of the polynomial modes which form the basis of the extended $\mathrm{GH}$ model (Theofilis 1997) elaborated upon herein; the eigenmodes discovered by Lin \& Malik (1996a) and analysed by Theofilis (1997) are the only three-dimensional instabilities relevant to the attachment-line region.

On the other hand, regarding subcriticality, Hall \& Malik (1986) have presented analysis and two-dimensional computations which delivered a bracket of Reynolds numbers $R e \in[535,583]$ where the flow could be subcritically destabilized. While Joslin (1995) and Balakumar (1998) asserted that nonlinear subcritical twodimensional equilibria were found in the DNS of the former and the secondary instability analysis of the latter investigator, the two-dimensional DNS of both Jiménez et al. (1990) and Theofilis (1998b), in which the GH structure is assumed, failed to find such solutions. In later works Joslin $(1996 a, 1997)$ suggested that on the one hand the treatment of disturbance pressure in Jiménez's code and on the other hand too low amplitudes of disturbances introduced in the nonlinear simulations of Theofilis $(1998 b)$ were responsible for this discrepancy.

Resolution of the issue of two-dimensional subcritical equilibria is of largely academic interest on two counts. Firstly, Hall \& Seddougui (1990) in the framework of a high-Reynolds-number approximation have analytically demonstrated that amplified three-dimensional instabilities may exist in this flow. While their analysis 
cannot be described exactly at finite Reynolds numbers, it further points to the necessity to clarify the role of three-dimensionality in the swept attachment-line boundary layer, including the potential of three-dimensionality to destroy subcritical nonlinear two-dimensional equilibria; the GH structure appears to be too restrictive for description of instability of the real flow. Secondly, two-dimensional nonlinear equilibria were reported as ceasing to exist below $R e \approx 535$ by Hall \& Malik (1986) and below $R e \approx 511$ by Balakumar (1998), leaving a very large parameter range $R e \in[245,511]$ (or $R e \in[245,535]$ ) unexplained; a different physical mechanism is required to fill either this gap or that with linear theory, $R e \in[245,583]$. In this respect, Joslin (1996a) postulated that interactions of multiple three-dimensional modes may lead to what he called 'bypass' transition.

The preceding discussion highlights that in order for further advances to be made in theory, new insight is necessary into, amongst other issues, the three-dimensional nature of the linear perturbations in the swept attachment-line boundary layer. To this end, Lin \& Malik (1996a) have explicitly stated that the GH mode, with its well-known linear dependence of the chordwise velocity component on the chordwise coordinate and independence of wall-normal and spanwise velocity components from that coordinate, is the only separable solution of the two-dimensional eigenvalue problem. It follows that one needs to solve the temporal two-dimensional partial derivative eigenvalue problem if information on the additional modes is required. Furthermore, if the point of view is taken that spatial linear stability results are directly amenable to comparisons with experiment, either the spatial two-dimensional linear stability problem must be solved at a yet higher computational cost compared with that of the already very demanding temporal two-dimensional eigenvalue problem (Heeg \& Geurts 1998) or spatial DNS must be performed in the linear regime, as done by Joslin (1996b), at a level of computational effort which is comparable with that of the spatial two-dimensional eigenvalue problem.

The present contribution shows that neither of the above approaches is necessary. Initially, the paper proceeds along two main themes: the partial derivative eigenvalue problem is solved using alternative numerical methods, more general boundary conditions (although symmetry/antisymmetry is expected) and a wider integration domain than that used by Lin \& Malik (1996a); the results of these authors (and only those) are recovered. Subsequently, the three-dimensional results of the partialderivative eigenvalue problem are analysed in the direction of flow acceleration by proposing an extension of the $\mathrm{GH}$ model in three spatial dimensions. It is demonstrated that the linear limit of the three-dimensional instability problem may be studied by solution of two systems of ordinary differential equations for symmetric and antisymmetric modes, respectively. Asymptotic analysis is next used in order to provide a multiple-scales perspective of the extended $\mathrm{GH}$ model; this analysis too provides an alternative algorithm for the determination of three-dimensional instability characteristics of this flow from solution of one-dimensional eigenvalue problems. The paper concludes by imposing the extended GH model eigenfunctions as initial conditions (at linearly low level) in three-dimensional spatial (nonlinear) DNS and monitoring the maintenance of the initial condition and the recovery of the imposed eigenvalues as part of the unsteady DNS results.

In $\S 2$ the basic flow model is introduced and the point at which the analysis of the results of Lin \& Malik (1996a) appeared to stagnate is discussed. In $\$ 3$ a numerical solution of the two-dimensional eigenvalue problem is presented and the spatial structure of the resulting eigenmodes, which gave rise to the introduction of the extended GH model, is discussed. The extended GH model is presented in $\S 4$, 
where all three-dimensional modes are classified into symmetric and antisymmetric disturbances; the systems of equations that these modes should respectively satisfy are presented. In $\$ 5.1$ the results of the extended GH model are compared to those of the temporal two-dimensional eigenvalue problem, while in $\$ 5.2$ the extended GH model is recast as a spatial linear stability eigenvalue problem, solved and its results are compared to those of the spatial two-dimensional eigenvalue problem and the spatial DNS of Joslin (1996b). The resolution of the apparent dispute on the form of disturbance pressure, introduced earlier, is presented in $\S 5.3$. Asymptotic analysis results and their connection with the extended GH model are presented in $\S 6$. The DNS work is presented in $\S 7$ and concluding remarks are furnished in $\S 8$.

\section{Linear instability in the attachment-line boundary layer in three spatial dimensions}

\subsection{The basic flow model}

We commence the presentation by a critical introduction of the basic flow model utilized herein, which is taken to be the swept Hiemenz boundary layer. $A$ priori one may justify the choice of this basic flow model based on the physically plausible assumptions used in its derivation; it models steady stagnation line flow in which the velocity components are independent of the homogeneous direction along the attachment line, $z$, which is assumed to be infinite, while all three basic flow velocity components are taken to depend on the wall-normal direction $y$. Moreover, the chordwise velocity component $\bar{U}$ is taken to be linearly dependent on the chordwise coordinate $x$, while the wall-normal velocity component $\bar{V}$ and the velocity component $\bar{W}$ along the attachment line are taken to be independent of $x$ (Rosenhead 1963). In the absence of a geometrical length scale in the flow acceleration direction, the properties of the velocity vector in the free stream are utilized. A length scale $\Delta=\sqrt{\nu / S}$ is constructed with the aid of the strain rate of the flow $S=\left(\mathrm{d} U_{e} / \mathrm{d} x\right)_{x=0}, v$ being the kinematic viscosity of the fluid and $U_{e}$ the chordwise component of velocity in the free stream. A Reynolds number $R e=W_{e} \Delta / \nu$, customarily denoted by $\bar{R}$, is then formed with the aid of the spanwise velocity component of the flow in the free stream, $W_{e}$. The degree of sweep compared with the oncoming flow direction is thus incorporated in the Reynolds number such that $R e=0$ corresponds to stagnationpoint flow, satisfying the classic unswept Hiemenz (1911) flow model. A system of ordinary differential equations for the determination of the swept Hiemenz basic-flow velocity components

$$
\bar{U}(x, y)=\frac{x}{\operatorname{Re}} \bar{u}(y), \quad \bar{V}(y)=\frac{1}{\operatorname{Re}} \bar{v}(y), \quad \bar{W}(y)=\bar{w}(y),
$$

is thus obtained,

$$
\begin{gathered}
\bar{u}+\bar{v}^{\prime}=0, \\
\bar{v}^{\prime \prime \prime}+\left(\bar{v}^{\prime}\right)^{2}-\overline{v v}^{\prime \prime}-1=0, \\
\bar{w}^{\prime \prime}-\overline{v w^{\prime}}=0,
\end{gathered}
$$

subject to the boundary conditions

$$
\bar{v}(0)=\kappa, \quad \bar{v}^{\prime}(0)=0, \quad \bar{v}^{\prime}(\infty)=-1, \quad \bar{w}(0)=0, \quad \bar{w}(\infty)=1,
$$

$\kappa$ being a non-dimensional parameter controlling suction at the wall. Aside from its 
mathematically attractive feature that it represents an exact solution of the steady incompressible Navier-Stokes equations, unlike the classic Blasius boundary layer, the swept Hiemenz boundary layer has been conclusively demonstrated to model well the basic flow in the physical problem at hand, at least as far back as the series of the experiments of Gaster (1967) and Poll (1979). Its limitations are encountered when curvature or compressibility are introduced into the physical problem. In both latter cases one has to rely on either matched asymptotic expansion solutions (Van Dyke 1975; Lin \& Malik 1996b) or computation.

An essential element of reliable stability analyses is the provision of accurate basic flow results. Here we solve for the swept Hiemenz basic flow numerically using Chebyshev spectral collocation. The $y$-momentum equation is of the FalknerSkan class, a problem which may be solved efficiently by Newton-Kantorowitz iteration (Boyd 1989). Spectral integration subsequently delivers the spanwise velocity component. The results are thus obtained on the same grid on which the stability analysis is performed and interpolation errors introduced from transferring data between grids are eliminated.

\subsection{Three-dimensional linear instability}

The introduction of the element of infinite span in the modelling of the physical problem permits considering the flow as homogeneous in this direction. Consequently an eigenmode ansatz may be introduced in $z$; given in addition that the temporal and spatial operators in the equations of motion are separable, disturbance quantities may be represented by

$$
\boldsymbol{q}(x, y, z, t)=\operatorname{Re}\left\{\boldsymbol{Q}_{p}(x, y) \exp [\mathrm{i}(\beta z-\Omega t)]\right\},
$$

where $Q_{p}(x, y)=(\hat{u}, \hat{v}, \hat{w}, \hat{p})^{\mathrm{T}}$ are complex two-dimensional amplitude functions of three-dimensional linear disturbances that are periodic in $z$. The dimensionality of the basic state has given rise to an instability analysis which is based on solution of a two-dimensional eigenvalue problem to be called BiGlobal instability analysis here, in order to differentiate it from other types of global analyses concerned with absolute instability of weakly non-parallel basic flows (Theofilis 2003). Unless otherwise stated in this paper the temporal concept has been utilized for linear instability, with $\beta$ a real wavenumber and $\Omega$ a complex eigenvalue. Physical significance is attached to $c_{\mathrm{r}}=\operatorname{Re}\{\Omega\} / \beta$ and $c_{\mathrm{i}}=\operatorname{Im}\{\Omega\} / \beta$, respectively denoting phase velocity and growth rate of the eigenmode. $\dagger$ From this point further discussion follows two distinct paths.

First, no further assumptions are made and the two-dimensional eigenvalue problem which results from substitution of (2.6) into the incompressible continuity and Navier-Stokes equations and linearization about the swept Hiemenz flow is solved numerically. This is the approach also followed by Lin \& Malik (1996a) who obtained BiGlobal eigenvalue problem results pertinent to boundary conditions of symmetry or antisymmetry. Here we have relaxed these conditions in order to introduce the possibility of additional solutions potentially existing in the problem appearing. In a brief statement attempting to analyse their results Lin \& Malik (1996a) asserted that

\footnotetext{
$\dagger$ The term 'wave' is avoided here since it stems from the form that infinitesimal disturbances assume when two out of three spatial directions are treated as homogeneous, e.g. in an infinite channel or the flat-plate boundary layer.
} 
only the Görtler-Hämmerlin mode † represents a truly separable solution that may be addressed by solution of the one-dimensional eigenvalue problem. Indeed, if one makes the assumption (Lin \& Malik 1996a)

$$
\boldsymbol{Q}_{p}(x, y)=\left(x^{m} \tilde{u}, x^{m-1} \tilde{v}, x^{m-1} \tilde{w}\right)^{\mathrm{T}}
$$

then only the GH mode is a separable solution of the disturbance equations. The reason is that, in conjunction with the swept Hiemenz basic flow, a factor $x^{m}$ remains in all but the streamwise viscous diffusion term in the streamwise momentum equation. In order for the system to be balanced at $O\left(x^{m}\right)$ linear dependence of $u(x, y)$ on $\hat{u}$ must be considered. From this discussion it followed that the partial derivative eigenvalue problem has to be solved for all eigenmodes additional to the GH.

As a second main theme of the present paper we introduce an analytical extension of the GH model in three spatial dimensions. The extended GH model proposed is based on the distinction between symmetric and antisymmetric modes, of which the mode discovered by Hall et al.(1984) in a temporal linear framework and fully confirmed by the DNS of Spalart (1988) and the spatial linear stability analysis of Theofilis (1995) is the first member. All additional two-dimensional modes discovered by Lin \& Malik (1996a) are recovered by the model proposed by solutions of one-dimensional linear eigenvalue problems. We present numerical solutions of the partial-derivative eigenvalue problem first and proceed to discuss the extended GH model thereafter.

\section{BiGlobal instability analysis; the partial-derivative linear eigenvalue problem}

For completeness the equations governing the two-dimensional eigenvalue problem solved are presented. A solution $Q(x, y, z, t)$ to the equations of motion is decomposed into

$$
\boldsymbol{Q}(x, y, z, t)=\boldsymbol{Q}_{b}(x, y)+\epsilon \operatorname{Re}\left\{\boldsymbol{Q}_{p}(x, y) \exp [\mathrm{i}(\beta z-\Omega t)]\right\},
$$

with $Q_{b}=(\bar{U}, \bar{V}, \bar{W}, \bar{P})^{T}$ indicating basic-flow velocity and pressure components. The decomposition is substituted into the incompressible continuity and Navier-Stokes equations. Linearization about the basic flow $\boldsymbol{Q}_{b}$ follows, based on the argument of smallness of the perturbation quantities. The basic-flow terms, themselves satisfying the equations of motion, are subtracted out. The following system of equations for the determination of $Q_{p}$ results:

$$
\begin{gathered}
\mathscr{D}_{x} \hat{u}+\mathscr{D}_{y} \hat{v}+\mathrm{i} \beta \hat{w}=0, \\
{\left[\mathcal{N}-\left(\mathscr{D}_{x} \bar{U}\right)\right] \hat{u}-\left(\mathscr{D}_{y} \bar{U}\right) \hat{v}-\mathscr{D}_{x} \hat{p}=-\mathrm{i} \Omega \hat{u},} \\
-\left(\mathscr{D}_{x} \bar{V}\right) \hat{u}+\left[\mathscr{N}-\left(\mathscr{D}_{y} \bar{V}\right)\right] \hat{v}-\mathscr{D}_{y} \hat{p}=-\mathrm{i} \Omega \hat{v}, \\
-\left(\mathscr{D}_{x} \bar{W}\right) \hat{u}-\left(\mathscr{D}_{y} \bar{W}\right) \hat{v}+\mathscr{N} \hat{w}-\mathrm{i} \beta \hat{p}=-\mathrm{i} \Omega \hat{w},
\end{gathered}
$$

where $\mathcal{N}=(1 / R e)\left(\mathscr{D}_{x}^{2}+\mathscr{D}_{y}^{2}-\beta^{2}\right)-\bar{U} \mathscr{D}_{x}-\bar{V} \mathscr{D}_{y}-\mathrm{i} \beta \bar{W}, \mathscr{D}_{x}=\partial / \partial x$, and $\mathscr{D}_{y}=\partial / \partial y$. The system is discretized in both the $x$ - and $y$-directions, resulting in the matrix eigenvalue problem

$$
\boldsymbol{A} X=\Omega \boldsymbol{B} X
$$

$\dagger$ Lin \& Malik (1996a) termed this mode 'HMP', after the authors who presented the first numerical solution of the corresponding one-dimensional eigenvalue problem; throughout we call the mode after the authors whose original ansatz was extended and utilized, GH for brevity. 
The system (3.2)-(3.5) is the most general system of equations which threedimensional, homogeneous in $z$, linear perturbations to the Navier-Stokes equations must satisfy. In classical boundary-layer stability theory the velocity component $\bar{V}$ in the $y$-direction is neglected and the problem is considered independent of the $x$ direction. An eigenmode ansatz is introduced to decompose flow quantities in Fourier modes in this spatial direction too, in a manner analogous to that introduced above for the $z$-direction. The system of equations resulting from these two assumptions which constitute what has come to be known as the 'parallel-flow approximation', may be combined into the well-known Orr-Sommerfeld equation which has been used for exhaustive stability investigations of a variety of incompressible flows (Drazin \& Reid 1981). The strong dependence of the basic flow on $x$ in the problem at hand does not permit introduction of eigenmodes in $x$, in general. However, in the framework of the swept Hiemenz model the basic-flow velocity vector satisfies (2.1), resulting in the elimination of the $x$-derivatives of the spanwise and normal basic-flow components from (3.2) (3.5). The interesting feature of the resulting stability problem is that if a structure analogous to (2.1) is considered for the disturbance velocity components, a one-dimensional eigenvalue problem is obtained. This observation, introduced by Görtler (1955) and Hämmerlin (1955) for reasons of mathematical convenience, was analysed by Hall et al.(1984) and utilized in their numerical solution of the eigenvalue problem which provided the first linear results which demonstrated close agreement with experiment.

We solve (3.2)-(3.5) presently without resort to the GH assumption but, rather, by resolving the chordwise and the wall-normal directions simultaneously. No-slip boundary conditions at the wall and $y \rightarrow \infty$, an accurate and validated extrapolation scheme at $|x| \rightarrow \infty$ (Theofilis, Hein \& Dallmann 2000) and pressure compatibility conditions complete the solution algorithm. Details on the numerical approach are presented elsewhere (Theofilis 1997, 2003); here we focus on the essential and fairly novel elements of our algorithm for the solution of the two-dimensional eigenvalue problem.

\subsection{Numerical methods}

As has also been mentioned by Lin \& Malik (1996a), obtaining solutions to the partial derivative eigenvalue problem is not trivial; thus the numerical methods utilized to recover the results presented deserve some discussion. The wall-normal direction is discretized using the same algorithm as that utilized for the solution of the basic flow problem, which is based on Chebyshev collocation. In the chordwise direction Jacobi collocation (of which the Chebyshev and Legendre polynomials are two members) is utilized, based on either the Gauss-Lobatto points or equidistant grids. By keeping the numerical method as close as possible to that of Lin \& Malik optimal conditions for comparisons are obtained while, at the same time, the availability of alternative algorithms provides the possibility to cross-validate results. It should be further noted that in order not to confine solutions to symmetric or antisymmetric disturbances we do not use collocation derivative matrices appropriate for such functions. Consequently we need twice as many collocation points as Lin \& Malik (1996a) in order to achieve results of comparable accuracy.

The main challenge encountered in two-dimensional linear stability solutions is that the size of the discretized eigenvalue problem (3.6) is such that current computer hardware technology is taken to its limits. Resolution of each spatial direction by typically upwards of 32 points results in a size of matrices $\boldsymbol{A}$ and $\boldsymbol{B}$ of the order of several hundreds of megabytes to gigabytes, when using the primitive-variables 
formulation. Moreover, if one utilizes a direct algorithm to recover the eigenspectrum, as done by Lin \& Malik (1996a), the runtime involved scales with the cube of the leading dimension of the discretized matrix and is of the order of CPU hours on a modern supercomputer. While the element of the size is introduced by the discretization, one may improve upon the speed of the computation. In our solution approach we use either the QZ algorithm or Arnoldi iteration (Saad 1980; Ehrenstein 1996) to recover the least stable part of the eigenspectrum of (3.6). The latter method proceeds by iteratively building a Krylov subspace of orthogonal vectors and an associated Hessenberg matrix. The eigenvalues of this matrix are an increasingly good approximation of those of the original problem as the dimension of the Krylov subspace increases. The efficiency of the Arnoldi iteration is obtained by the fact that the size of the Hessenberg matrix which delivers accurate eigenvalues of the original problem is a small fraction of the size of $\boldsymbol{A}$ and $\boldsymbol{B}$. Order-of-magnitude improvement may thus be obtained in terms of runtime compared with the QZ algorithm.

An additional benefit of using Krylov subspace iteration is offered by the possibility to solve the shifted-and-inverted problem

$$
\hat{\boldsymbol{A}} X=\mu X, \quad \hat{\boldsymbol{A}}=(\boldsymbol{A}-\sigma \boldsymbol{B})^{-1} \boldsymbol{B}, \quad \mu=\frac{1}{\Omega-\sigma},
$$

instead of (3.6). Here $\sigma$ represents an estimate of a desired eigenvalue, the neighbourhood of which is resolved by the Arnoldi iteration. Taking advantage of the sparsity of $\boldsymbol{B}$, only matrix $\hat{\boldsymbol{A}}$ need be stored. By contrast, if the QZ algorithm is used, both matrices of the original problem and two additional matrices of equal size, containing the eigenvectors, are required. The ability to store one rather than four matrices of large size results in the possibility of solving substantially larger (in terms of resolution) problems when using the Arnoldi iteration than would be possible by the straightforward QZ algorithm. With all considerations taken into account, the highest resolution results presented represent the limit of our computing facilities, a fact which further underlines the significance of the analysis based on the extended GH model presented in $\S 4$.

\subsection{BiGlobal linear stability results}

We focus on the only temporal results available for quantitative comparisons, presented by Lin \& Malik (1996a), at $R e=800, \beta=0.255$. In taking our calculations as far as possible in the chordwise direction we obtain solutions in $x \in[-150,150] \times$ $y \in[0,100]$ in non-dimensional units. Such a domain is approximately equal in the wall-normal and three times as large in the chordwise directions compared with that of Lin \& Malik (1996a). Our grid sequencing for the eigenvalues of the first four (and as results show the only) unstable modes at the parameters chosen is presented in table 1.

It may be seen that the two-dimensional (in $x$ and $y$ ) analogue of the GörtlerHämmerlin (GH) mode, which is solved for in $y$ and prescribed in $x$ in the framework of the linear theory of Hall et al. (1984), is present as a result of the partial-derivative eigenvalue problem. Moreover, the chordwise resolution does not affect the accuracy with which this mode is captured. On the other hand, turning to the less amplified linear modes, A1, S2 and A2, in the nomenclature of Lin \& Malik (1996a), and in full agreement with the results of these authors, we find an influence of the chordwise resolution on the accuracy of the modes obtained. Interestingly, the mode requiring the least number of nodes to resolve is mode $\mathrm{A} 1$, while a progressively larger number 
Resolution

$$
\begin{gathered}
8 \times 48 \\
16 \times 48 \\
24 \times 48 \\
32 \times 48 \\
\text { LM }
\end{gathered}
$$

$8 \times 48$
$16 \times 48$
$24 \times 48$
$32 \times 48$
LM

\begin{tabular}{ll}
\hline 0.35840980 & 0.00585327 \\
0.35840980 & 0.00585327 \\
0.35840980 & 0.00585327 \\
0.35840980 & 0.00585327 \\
0.35840982 & 0.00585325
\end{tabular}

S2

\begin{tabular}{ll}
\hline 0.35767778 & 0.00267928 \\
0.35743957 & 0.00233906 \\
0.35743454 & 0.00234015 \\
0.35743981 & 0.00234523 \\
0.35743540 & 0.00234300
\end{tabular}

A1

\begin{tabular}{ll}
\hline 0.35788801 & 0.00407816 \\
0.35791950 & 0.00409899 \\
0.35791952 & 0.00409918 \\
0.35791953 & 0.00409872 \\
0.35791970 & 0.00409887
\end{tabular}

A2

$\begin{array}{ll}0.35757403 & 0.00141244 \\ 0.35699469 & 0.00054502 \\ 0.35696398 & 0.00057615 \\ 0.35694358 & 0.00058446 \\ 0.35695687 & 0.00058571\end{array}$

TABLE 1. Grid refinement history in the numerical solution of the two-dimensional eigenvalue problem at $\kappa=0, R e=800, \beta=0.255$. Also presented, denoted by 'LM', are the results of Lin \& Malik (1996a).

of collocation points is required to resolve modes S2 and A2. This observation will be clarified in the context of the analysis of the linear stability results which follows in $\$ 4$.

The agreement of the results presented herein with those of Lin \& Malik (1996a) ranges from excellent for the $\mathrm{GH}$ mode to very good for mode $\mathrm{A} 2$, the least significant from a stability analysis point of view. In all cases the discrepancies are confined in the sixth, or higher, decimal place, translating to a relative discrepancy of frequencies and growth rates of $O(0.01-0.1 \%)$. Factors such as the Krylov subspace dimension or the linear extrapolation boundary condition at the location where the domain was truncated at large chordwise distances from the attachment line may be considered responsible for the residual discrepancy. In what follows it is demonstrated that, using the same number of points to resolve the $x$-direction, convergence is reached faster in the results of Lin \& Malik (1996a) compared with our results presented in table 1. This is to be expected for two reasons: firstly we resolve a much larger domain than these authors do and secondly, we do not impose any symmetries on the solutions expected. When we confine our attention to the domain used by Lin \& Malik (1996a) and utilize collocation derivative matrices appropriate for symmetric/antisymmetric modes the agreement improves significantly. However, rather than imposing symmetry for the sake of optimal comparison, we prefer to present the results of table 1, which demonstrate that the imposition of symmetries in the problem at hand, as done by Lin \& Malik (1996a), delivers identical results with those obtained without symmetries imposed in the solutions sought.

Another interesting observation concerns the frequencies of the modes which exist additionally to the $\mathrm{GH}$ mode in a three-dimensional attachment-line boundary layer. In the comparisons between theory and experiment presented by Theofilis (1998a) it was puzzling that a rather large error bar existed in the experimental results for the frequency (the only available result) of unstable eigenmodes. While it is certainly not trivial to perform high-Reynolds-number experiments in the swept attachment-line boundary layer, the support of the frequency functions shown in the experiments of Poll et al. (1996) is rather large and different from the substantially cleaner spectra 
obtained, e.g. in the Blasius boundary layer. The closeness of the frequencies of modes $\mathrm{A} 1, \mathrm{~S} 2, \ldots$ with that of what was thought to be a pure GH growing disturbance, may provide an explanation for this observation. In the three-dimensional environment in which the experiments are performed, the additional modes will always be present alongside the GH mode at frequencies very close to that of the latter mode. The experimental information that may separate the least-stable GH mode from the rest is on the growth rate, which no experiment to date has provided.

\subsection{The spatial structure of the two-dimensional eigenfunctions}

In presenting their BiGlobal linear stability results, Lin \& Malik (1996a) confined themselves to asserting that the spanwise and wall-normal velocity components of the GH mode show absolutely no variation in the chordwise direction, while the chordwise velocity component of this mode and all disturbance eigenfunctions of the new modes discovered grow in $x$. Although this assertion is demonstrated in their normalized one-dimensional eigenfunctions, and is fully verified in our results, it is impossible to quantify the growth of the eigenmodes in $x$ from the results that Lin \& Malik (1996a) presented.

While the introduction of an additional spatial dimension complicates graphical presentation of results, the essential features of the spatial structure of the eigenfunctions may be visualized by a perspective view of the results, such as that presented in figures 1-3. In part $(a)$ of all three figures the real parts of the twodimensional disturbance velocity components are presented, normalized by their respective maxima. In parts $(b)$ and $(c)$ of the figures we present the dependence of the disturbance eigenfunctions on the chordwise coordinate at two $y$ locations, $(b)$ one very close to the wall $y_{1} \approx 0.04$ and $(c)$ one further out inside the boundary layer, $y_{2} \approx 2$. Approximately half of the collocation nodes are within $y<y_{2}$ in the wallnormal direction. In parts $(b)$ and $(c)$ of the figures both the real and the imaginary parts of the disturbance eigenfunctions are presented. A number of observations on these figures deserve thorough discussion.

In figure 1 one may find proof that the assumption made by Görtler and Hämmerlin for mathematical convenience is one which corresponds to the physics of the threedimensional attachment-line boundary layer. The dependence of $\hat{u}(x, y)$ on $x$ is clearly linear, while $\partial \hat{v}(x, y) / \partial x=\partial \hat{w}(x, y) / \partial x=0$. This three-dimensional analogue of the $\mathrm{GH}$ mode possesses the highest growth rate or lowest damping rate at all Reynolds numbers, a fact which is in line with one of the most significant results of the spatial direct numerical simulation of Spalart (1988), namely that in three-dimensional attachment-line boundary layer flow an instability mode possessing the symmetry imposed by the GH ansatz is that which grows fastest in a linear framework. Further evidence that the GH mode is indeed the most significant from a linear stability analysis point of view may be found in the comparisons of Theofilis (1998b) between on the one hand results of one-dimensional eigenvalue problems and two-dimensional temporal DNS in which the GH assumption is incorporated, and experimental results (Poll et al.1996) on the other. The results of the two-dimensional eigenvalue problem, though, in which the $x$-direction is fully resolved and no symmetry of the mode is expected or imposed, constitute a firm demonstration of the validity of the GH assumption.

Inspection of the results of modes $\mathrm{A} 1$ and $\mathrm{S} 2$, the next in significance, from a stability analysis point of view, delivers essential new information which is absent from the work of Lin \& Malik (1996a) and is intimately related with the theoretical model discussed in the next section. In figure 2 the spatial structure of the velocity 
$\operatorname{Re}(u) \quad \operatorname{Re}(v) \quad \operatorname{Re}(w)$

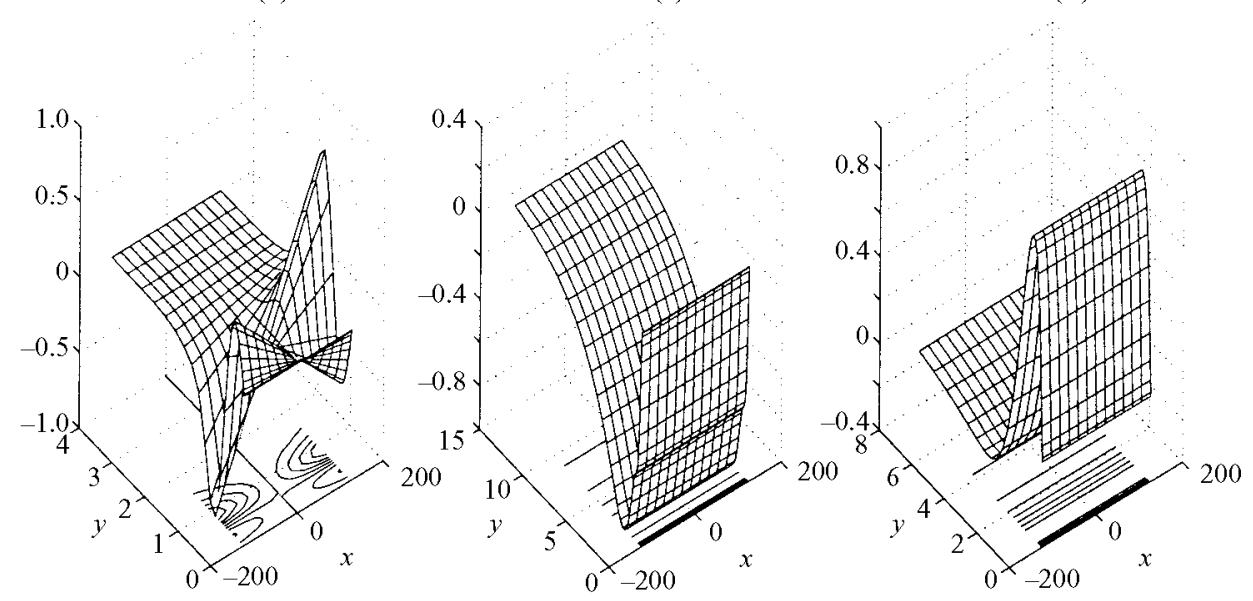

(b)
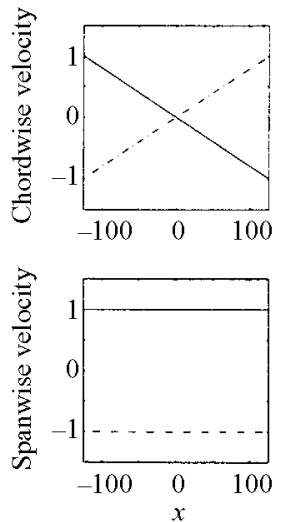
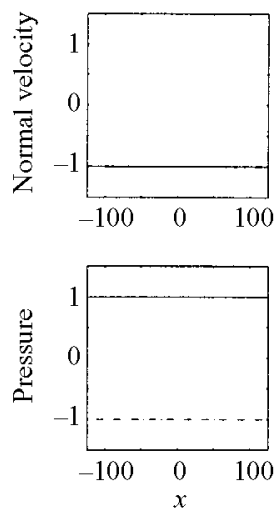

(c)
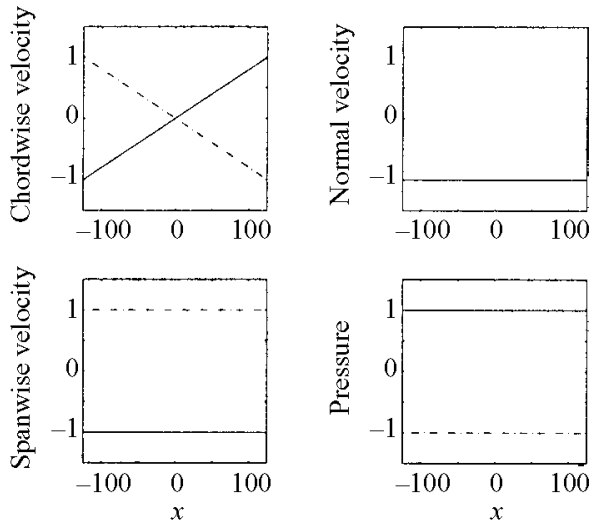

FIgure 1. (a) Perspective view of the real parts of the disturbance eigenfunctions of the Görtler-Hämmerlin (GH/S1) linear eigenmode, as obtained by numerical solution of the partial derivative eigenvalue problem $(3.2)-(3.5)$. $(b, c)$ The dependence of the disturbance eigenfunctions $\hat{q}$ on $x$ at $y_{1} \approx 0.04(b)$ and $y_{2} \approx 2(c) ;$ solid $\operatorname{Re}\{\hat{q}\}$, dashed $\operatorname{Im}\{\hat{q}\}$.

disturbances of mode A1 is presented. In figure 2(a), one may obtain the analogues of the one-dimensional plots presented by Lin \& Malik (1996a) by monitoring the $y$ dependence of the eigenfunctions at constant $x$. Most significantly, however, it is conceivable that the far-field (in $x)$ dependence of $\hat{u}(x, y)$ on $x$ at constant $y$ is quadratic, albeit at clearly different values of the parameters pertinent to the parabolas at each $y$ location. Analogously, the dependence of $\hat{v}(x, y), \hat{w}(x, y)$ and $\hat{p}(x, y)$ on $x$ at constant $y$ is linear, again slopes being a function of $y$. This structure is clearer in figure $2(b, c)$. A qualitatively analogous observation is made in the results for mode $\mathrm{S} 2$, presented in figure 3 . Here $\hat{u}(x, y)$ appears to be a cubic function of $x$, with coefficients functions of $y$, while $\hat{v}(x, y), \hat{w}(x, y)$ and $\hat{p}(x, y)$ appear to depend 
(a)
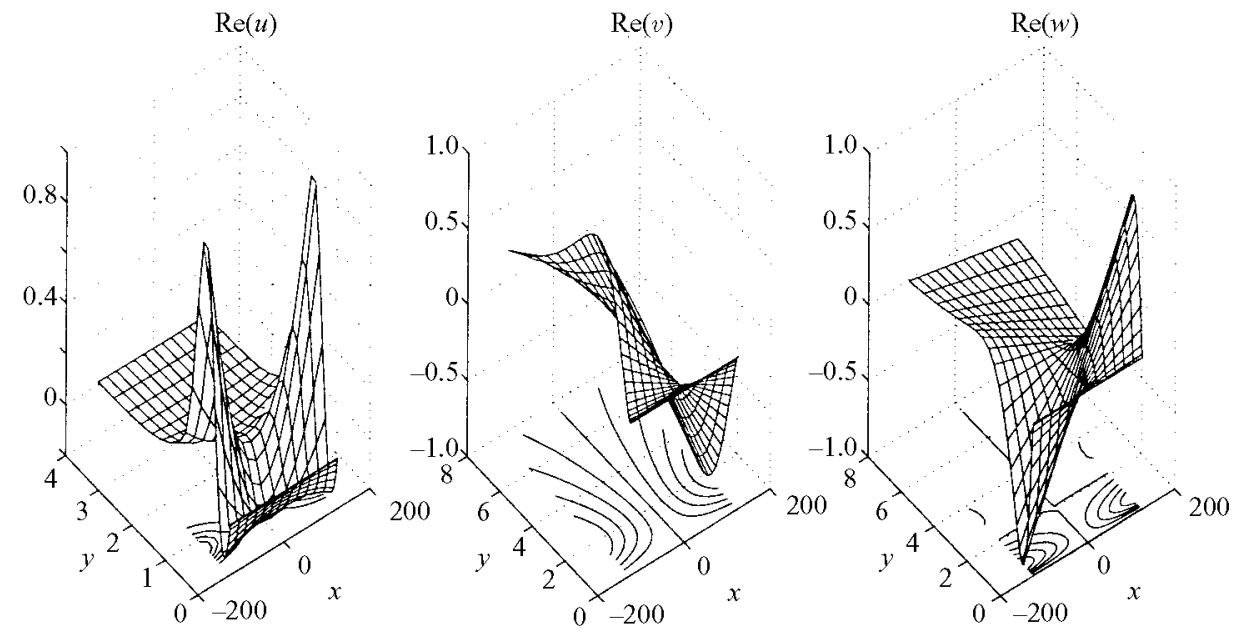

(b)
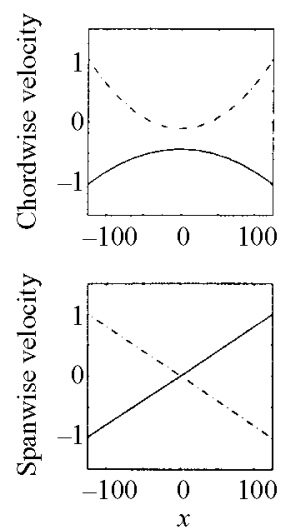
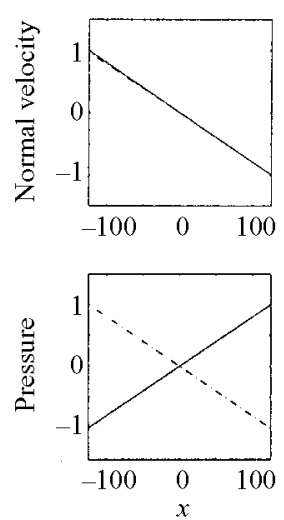

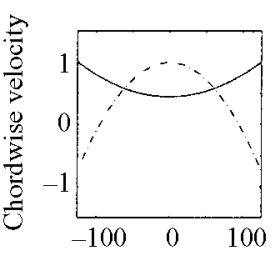

(c)
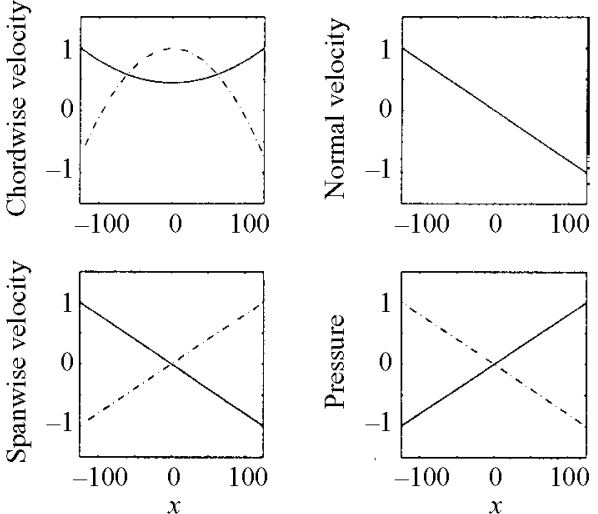

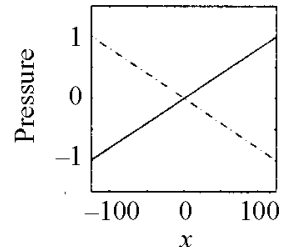

FiguRE 2. (a) Perspective view of the real parts of the disturbance eigenfunctions of the linear eigenmode A1, as obtained by numerical solution of the partial derivative eigenvalue problem (3.2)-(3.5). (b, c) The dependence of the disturbance eigenfunctions $\hat{q}$ on $x$ at $y_{1} \approx 0.04$ (b) and $y_{2} \approx 2(c)$; solid $\operatorname{Re}\{\hat{q}\}$, dashed $\operatorname{Im}\{\hat{q}\}$.

quadratically on $x$ at a fixed $y$ location. Higher modes are not presented, since they have been found to follow an analogous pattern, namely eigenfunctions for $\hat{u}$ appear as powers of $x$ one higher than those on which $\hat{v}, \hat{w}$ and $\hat{p}$ seemingly depend.

At this point the structure (2.7) may be introduced and fail on the grounds discussed. In the next section we present the extended GH model which successfully unravels the $x$-dependence of the eigenfunctions and delivers their eigenvalues by solving onedimensional eigenvalue problems. Note that the increasingly steeper dependence of the two-dimensional eigenfunctions on $x$ as $|x| \rightarrow \infty$ may well be connected with the convergence difficulties for the higher modes at low chordwise resolution, discussed 
$\operatorname{Re}(u) \quad \operatorname{Re}(v) \quad \operatorname{Re}(w)$

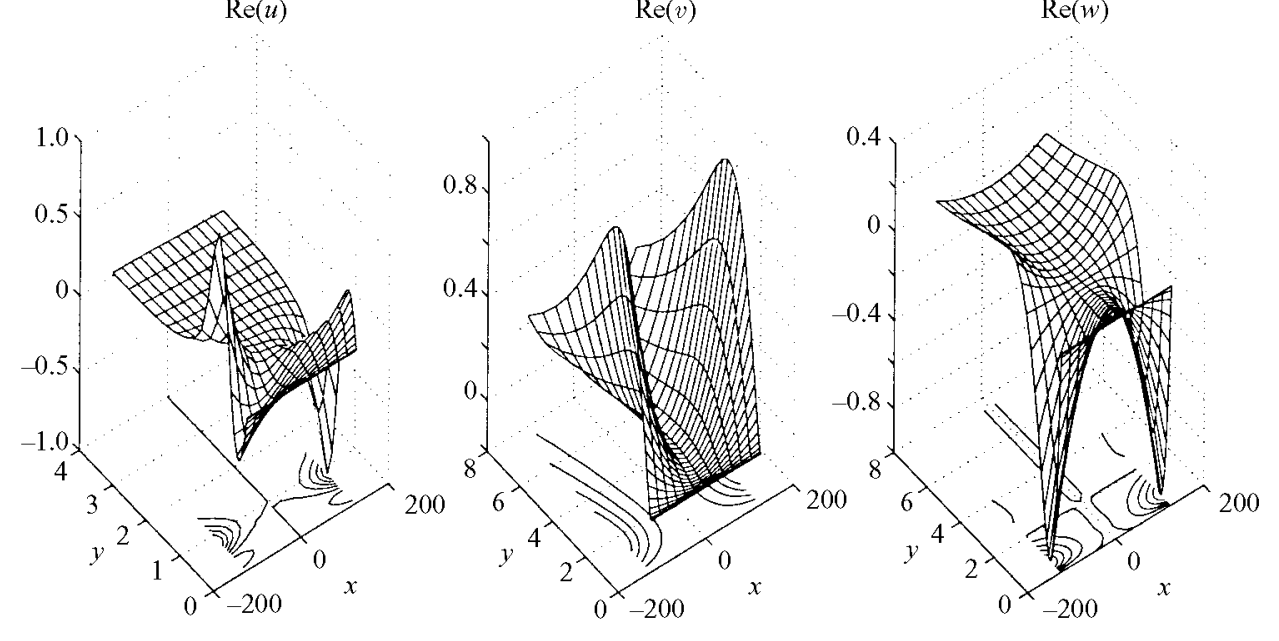

(b)
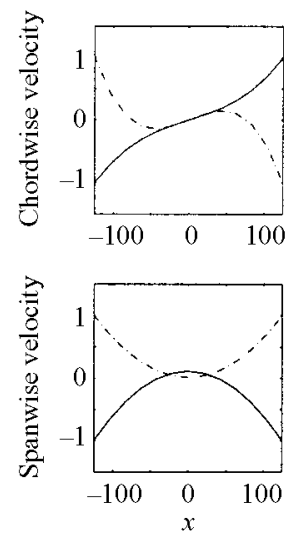
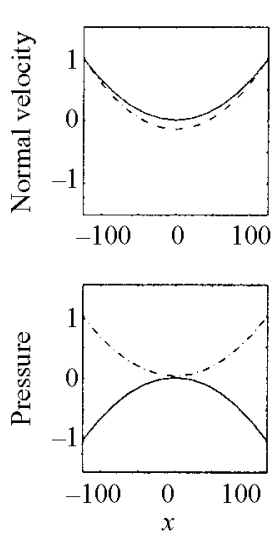

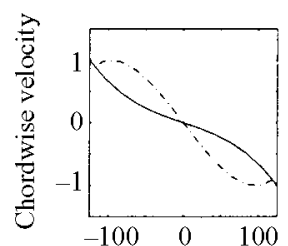

(c)
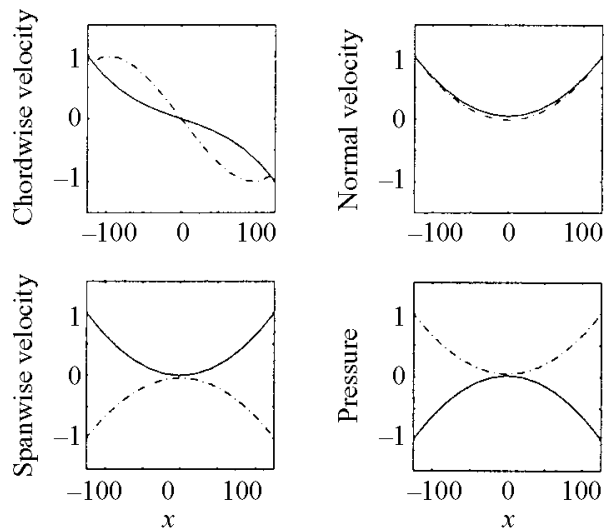

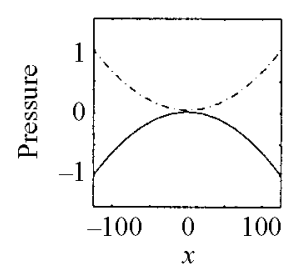

FIgURE 3. (a) Perspective view of the real parts of the disturbance eigenfunctions of the linear eigenmode $\mathrm{S} 2$, as obtained by numerical solution of the partial derivative eigenvalue problem (3.2)-(3.5). (b, c) The dependence of the disturbance eigenfunctions $\hat{q}$ on $x$ at $y_{1} \approx 0.04$ (b) and $y_{2} \approx 2(c)$; solid $\operatorname{Re}\{\hat{q}\}$, dashed $\operatorname{Im}\{\hat{q}\}$.

in the previous section, if no prior knowledge of the behaviour of the eigenfunctions in the chordwise direction is incorporated in the solution algorithm.

\section{Extension of the Görtler-Hämmerlin model to three dimensions}

The deficiency of the simple model (2.7) may be remedied in a straightforward manner by extending the Görtler-Hämmerlin (1955) ansatz to a model which classifies all linear three-dimensional disturbances in the incompressible swept attachment-line boundary layer into two classes: 
symmetric modes, $(\hat{u}, \hat{v}, \hat{w})^{\mathrm{T}}$, satisfying

$$
\left.\begin{array}{c}
\hat{u}(x, y)=\sum_{m=1}^{M} x^{2 m-1} \tilde{u}_{2 m-1}(y), \\
\hat{v}(x, y)=\sum_{m=1}^{M} x^{2 m-2} \tilde{v}_{2 m-2}(y), \\
\hat{w}(x, y)=\sum_{m=1}^{M} x^{2 m-2} \tilde{w}_{2 m-2}(y),
\end{array}\right\}
$$

and antisymmetric modes, $(\hat{u}, \hat{v}, \hat{w})^{\mathrm{T}}$, which satisfy

$$
\left.\begin{array}{rl}
\hat{u}(x, y) & =\sum_{m=0}^{M} x^{2 m} \tilde{u}_{2 m}(y), \\
\hat{v}(x, y) & =\sum_{m=1}^{M} x^{2 m-1} \tilde{v}_{2 m-1}(y), \\
\hat{w}(x, y) & =\sum_{m=1}^{M} x^{2 m-1} \tilde{w}_{2 m-1}(y) .
\end{array}\right\}
$$

After truncation at some $M \geqslant 1$, substitution into the incompressible continuity and Navier-Stokes equations and linearization, one may solve the resulting system directly or address the eigenvalue problem defined by the highest-order terms retained in the expansion independently from those pertaining to lower powers of $x$.

\subsection{Derivation of the systems governing three-dimensional linear instability}

\subsubsection{Symmetric modes}

For symmetric modes the structure

$$
\begin{aligned}
u & =\frac{x}{R e} \bar{u}+\epsilon \hat{u}(x, y) \mathrm{e}^{\mathrm{i}(\beta z-\Omega t)}, \\
v & =\frac{1}{R e} \bar{v}+\epsilon \hat{v}(x, y) \mathrm{e}^{\mathrm{i}(\beta z-\Omega t)}, \\
w & =\bar{w}+\epsilon \hat{w}(x, y) \mathrm{e}^{\mathrm{i}(\beta z-\Omega t)},
\end{aligned}
$$

with $(\hat{u}, \hat{v}, \hat{w})^{\mathrm{T}}$ satisfying (4.1) and $\epsilon \ll 1$ is substituted into the incompressible continuity and Navier-Stokes equations. Linearization based on the smallness of $\epsilon$ follows; in order for the resulting systems to be closed at $O(\epsilon)$ and a given power of $x^{m}$, the disturbance pressure must satisfy constraints discussed in what follows. The situation is illustrated by reference to the systems pertinent to $M=1,2$ and 3 ; these are

System S1:

$$
\begin{gathered}
\tilde{u}_{1}+\tilde{v}_{0}^{\prime}+\mathrm{i} \beta \tilde{w}_{0}=0, \\
\{\mathscr{L}-2 \bar{u}\} \tilde{u}_{1}-\bar{u}^{\prime} \tilde{v}_{0}+6 \tilde{u}_{3}-\operatorname{Re} \tilde{p}_{2}=-\mathrm{i} \Omega \operatorname{Re} \tilde{u}_{1}, \\
\left\{\mathscr{L}-\bar{v}^{\prime}\right\} \tilde{v}_{0}-\operatorname{Re} \tilde{p}_{0}^{\prime}+2 \tilde{v}_{2}=-\mathrm{i} \Omega \operatorname{Re} \tilde{v}_{0}, \\
-\operatorname{Re} \bar{w}^{\prime} \tilde{v}_{0}+\mathscr{L} \tilde{w}_{0}-\mathrm{i} \beta \operatorname{Re} \tilde{p}_{0}+2 \tilde{w}_{2}=-\mathrm{i} \Omega \operatorname{Re} \tilde{w}_{0}
\end{gathered}
$$

System S2:

$$
\begin{gathered}
3 \tilde{u}_{3}+\tilde{v}_{2}^{\prime}+\mathrm{i} \beta \tilde{w}_{2}=0, \\
\{\mathscr{L}-4 \bar{u}\} \tilde{u}_{3}-\bar{u}^{\prime} \tilde{v}_{2}+20 \tilde{u}_{5}-\operatorname{Re} \tilde{p}_{4}=-\mathrm{i} \Omega \operatorname{Re} \tilde{u}_{3},
\end{gathered}
$$




$$
\begin{gathered}
\left\{\mathscr{L}-\bar{v}^{\prime}-2 \bar{u}\right\} \tilde{v}_{2}-\frac{1}{2} \operatorname{Re} \tilde{p}_{2}^{\prime}+12 \tilde{v}_{4}=-\mathrm{i} \Omega \operatorname{Re} \tilde{v}_{2}, \\
-\operatorname{Re} \bar{w}^{\prime} \tilde{v}_{2}+\{\mathscr{L}-2 \bar{u}\} \tilde{w}_{2}-\frac{1}{2} \operatorname{Re} \beta \beta \tilde{p}_{2}+12 \tilde{w}_{4}=-\mathrm{i} \Omega \operatorname{Re} \tilde{w}_{2} ;
\end{gathered}
$$

System S3:

$$
\begin{gathered}
5 \tilde{u}_{5}+\tilde{v}_{4}^{\prime}+\mathrm{i} \beta \tilde{w}_{4}=0, \\
\{\mathscr{L}-6 \bar{u}\} \tilde{u}_{5}-\bar{u}^{\prime} \tilde{v}_{4}=-\mathrm{i} \Omega \operatorname{Re} \tilde{u}_{5}, \\
\left\{\mathscr{L}-\bar{v}^{\prime}-4 \bar{u}\right\} \tilde{v}_{4}-\frac{1}{4} \operatorname{Re} \tilde{p}_{4}^{\prime}=-\mathrm{i} \Omega \operatorname{Re} \tilde{v}_{4}, \\
-\operatorname{Re} \bar{w}^{\prime} \tilde{v}_{4}+\{\mathscr{L}-4 \bar{u}\} \tilde{w}_{4}-\frac{1}{4} \operatorname{Rei} \beta \tilde{p}_{4}=-\mathrm{i} \Omega \operatorname{Re} \tilde{w}_{4} ;
\end{gathered}
$$

where

$$
\mathscr{L}=\mathscr{D}^{2}-\bar{v} \mathscr{D}-\beta^{2}-\mathrm{i} \beta R e \bar{w},
$$

$\mathscr{D} \equiv \mathrm{d} / \mathrm{d} y$, and $\mathscr{D}^{2} \equiv \mathrm{d}^{2} / \mathrm{d} y^{2}$. At $M=1$ the system pertinent to the GH ansatz (Hall et al. 1984) is recovered. If $M=2$, on the other hand, two effects come into play. First, the velocity components $\left(\tilde{u}_{3}, \tilde{v}_{2}, \tilde{w}_{2}\right)^{\mathrm{T}}$ modify the original system. Most significantly, $\tilde{u}_{3}$ accounts for the inability of the simple model (2.7) proposed by Lin \& Malik (1996a) to close the system at $O(x)$. Second, on account of the $x$-momentum equation, the disturbance pressure may take the form

$$
\hat{p}=\left[\tilde{p}_{0}+\frac{1}{2} x^{2} \tilde{p}_{2}+\frac{1}{4} x^{4} \tilde{p}_{4}\right] \mathrm{e}^{\mathrm{i}(\beta z-\Omega t)},
$$

so that both linear and cubic terms in $x$ are balanced. However, on account of the $y$-momentum equation, the quartic pressure term must either vanish or be absorbed into the terms of lower-order in $x$, in order for the equations to be balanced at $O\left(x^{0}\right)$ and $O\left(x^{2}\right)$. Specifically,

$$
\frac{1}{4} x^{4} \tilde{p}_{4}^{\prime}=\left\{\begin{array}{l}
F(y) x^{2} \\
G(y) \\
0 .
\end{array}\right.
$$

Either of the first two possibilities may be absorbed into the structure

$$
\hat{p}=\left[\tilde{p}_{0}+\frac{1}{2} x^{2} \tilde{p}_{2}\right] \mathrm{e}^{\mathrm{i}(\beta z-\Omega t)}
$$

by suitable redefinition of $\tilde{p}_{0}$ and $\tilde{p}_{2}$; equation (4.20) is therefore used for the pressure perturbation at $M=2$. The argument is carried forward to all higher values of $M$. This results in an eigenvalue problem for the highest-order terms retained in the expansion which may be solved independently of those pertaining to lower expansion coefficients. The pattern emerging at the highest power of $x$ and a given truncation $M$ is

$$
\begin{gathered}
(2 M-1) \hat{u}_{2 M-1}+\hat{v}_{2 M-2}^{\prime}+\mathrm{i} \beta \hat{w}_{2 M-2}=0, \\
\{\mathscr{L}-2 M \bar{u}\} \hat{u}_{2 M-1}-\bar{u}^{\prime} \hat{v}_{2 M-2}=-\mathrm{i} \Omega \operatorname{Re} \hat{u}_{2 M-1}, \\
\left\{\mathscr{L}-\bar{v}^{\prime}-(2 M-2) \bar{u}\right\} \hat{v}_{2 M-2}-\frac{1}{2 \mathrm{M}-2} \operatorname{Re} \hat{p}_{2 M-2}^{\prime}=-\mathrm{i} \Omega \operatorname{Re} \hat{v}_{2 M-2}, \\
-\operatorname{Re} \bar{w}^{\prime} \hat{v}_{2 M-2}+\{\mathscr{L}-(2 M-2) \bar{u}\} \hat{w}_{2 M-2}-\frac{1}{2 \mathrm{M}-2} \mathrm{i} \beta \operatorname{Re} \hat{p}_{2 M-2}=-\mathrm{i} \Omega \operatorname{Re} \hat{w}_{2 M-2} .
\end{gathered}
$$

The factor unity replaces $1 /(2 M-2)$ in multiplying the disturbance pressure at $M=1$. Solution of (4.21)-(4.24) delivers a single most-unstable or least-damped eigenmode. We identify this mode by the value of $M$ at which truncation is performed, S1 for the GH mode, S2 for the mode pertinent to $M=2$ and so on. 


\subsubsection{Antisymmetric modes}

In an analogous manner, the structure (4.3)-(4.5) is substituted into the incompressible continuity and Navier-Stokes equations with $(\hat{u}, \hat{v}, \hat{w})^{\mathrm{T}}$ taken to satisfy (4.2). Again $\epsilon$ is taken to be small for linearization to be permissible and, following an analogous reasoning to that for symmetric disturbances, antisymmetric pressure eigenmodes are taken to assume the form

$$
\hat{p}=\left[x \tilde{p}_{1}+\frac{1}{3} x^{3} \tilde{p}_{3}\right] \mathrm{e}^{\mathrm{i}(\beta z-\Omega t)}
$$

such that, for example, at $M=2$ one obtains

Equation A0:

$$
\{\mathscr{L}-\bar{u}\} \tilde{u}_{0}+2 \tilde{u}_{2}-\operatorname{Re} \tilde{p}_{1}=-\mathrm{i} \Omega \operatorname{Re} \tilde{u}_{0}
$$

System A1:

$$
\begin{gathered}
2 \tilde{u}_{2}+\tilde{v}_{1}^{\prime}+\mathrm{i} \beta \tilde{w}_{1}=0, \\
\{\mathscr{L}-3 \bar{u}\} \tilde{u}_{2}-\bar{u}^{\prime} \tilde{v}_{1}+12 \tilde{u}_{4}-\operatorname{Re} \tilde{p}_{3}=-\mathrm{i} \Omega \operatorname{Re} \tilde{u}_{2}, \\
\left\{\mathscr{L}-\bar{u}-\bar{v}^{\prime}\right\} \tilde{v}_{1}-\operatorname{Re} \tilde{p}_{1}^{\prime}+6 \tilde{v}_{3}=-\mathrm{i} \Omega \operatorname{Re} \tilde{v}_{1}, \\
-\operatorname{Re} \bar{w}^{\prime} \tilde{v}_{1}+\{\mathscr{L}-\bar{u}\} \tilde{w}_{1}-\mathrm{i} \beta \operatorname{Re} \tilde{p}_{1}+6 \tilde{w}_{3}=-\mathrm{i} \Omega \operatorname{Re} \tilde{w}_{1} ;
\end{gathered}
$$

System A2:

$$
\begin{gathered}
4 \tilde{u}_{4}+\tilde{v}_{3}^{\prime}+\mathrm{i} \beta \tilde{w}_{3}=0, \\
\{\mathscr{L}-5 \bar{u}\} \tilde{u}_{4}-\bar{u}^{\prime} \tilde{v}_{3}=-\mathrm{i} \Omega \operatorname{Re} \tilde{u}_{4}, \\
\left\{\mathscr{L}-3 \bar{u}-\bar{v}^{\prime}\right\} \tilde{v}_{3}-\frac{1}{3} \operatorname{Re} \tilde{p}_{3}^{\prime}=-\mathrm{i} \Omega \operatorname{Re} \tilde{v}_{3}, \\
-\operatorname{Re} \bar{w}^{\prime} \tilde{v}_{3}+\{\mathscr{L}-3 \bar{u}\} \tilde{w}_{3}-\frac{1}{3} \mathrm{i} \beta \operatorname{Re} \tilde{p}_{3}=-\mathrm{i} \Omega \operatorname{Re} \tilde{w}_{3} ;
\end{gathered}
$$

leading, at a given truncation parameter $M$ and highest-order, to the system

$$
\begin{gathered}
2 M \hat{u}_{2 M}+\hat{v}_{2 M-1}^{\prime}+\mathrm{i} \beta \hat{w}_{2 M-1}=0, \\
\{\mathscr{L}-(2 M+1) \bar{u}\} \hat{u}_{2 M}-\bar{u}^{\prime} \hat{v}_{2 M-1}=-\mathrm{i} \Omega \operatorname{Re} \hat{u}_{2 M}, \\
\left\{\mathscr{L}-\bar{v}^{\prime}-(2 M-1) \bar{u}\right\} \hat{v}_{2 M-1}-\frac{1}{2 \mathrm{M}-1} \operatorname{Re} \hat{p}_{2 M-1}^{\prime}=-\mathrm{i} \Omega \operatorname{Re} \hat{v}_{2 M-1}, \\
-\operatorname{Re} \bar{w}^{\prime} \hat{v}_{2 M-1}+\{\mathscr{L}-(2 M-1) \bar{u}\} \hat{w}_{2 M-1}-\frac{1}{2 \mathrm{M}-1} \mathrm{i} \beta \operatorname{Re} \hat{p}_{2 M-1}=-\mathrm{i} \Omega \operatorname{Re} \hat{w}_{2 M-1} .
\end{gathered}
$$

In either case of system (4.21)-(4.24) or (4.35)-(4.38) boundary conditions are imposed on disturbance velocity components alone, viscous boundary conditions at the wall, homogeneous Dirichlet conditions in the far field and vanishing of the first derivative of the wall-normal disturbance velocity components at both ends of the respective integration domains.

\subsection{Extension of the Görtler-Hämmerlin (1955) model to three-dimensional disturbances}

Any of the four disturbance eigenfunctions appearing in the systems (4.21)-(4.24) and (4.35)-(4.38) may be eliminated in order to arrive at a more compact form of the systems governing symmetric and antisymmetric disturbances, $\mathrm{S}$ and $\mathrm{A}$, respectively. 
Defining the linear operator

$$
\begin{aligned}
\mathscr{M}=\mathscr{D}^{4}-\bar{v} \mathscr{D}^{3}-\left[2(M-1) \bar{u}+\bar{v}^{\prime}+\mathrm{i} \beta R e \bar{w}+2 \beta^{2}\right] \mathscr{D}^{2}+\left[\bar{u}^{\prime}+\beta^{2} \bar{v}\right] \mathscr{D} \\
+2(M-1) \beta^{2} \bar{u}+\beta^{2} \bar{v}^{\prime}+\beta^{4}+\mathrm{i} \beta^{3} R e \bar{w}+\mathrm{i} \beta R e \bar{w}^{\prime \prime},
\end{aligned}
$$

the systems which the symmetric, $\tilde{u}_{2 m-1}, \tilde{v}_{2 m-2}$, and antisymmetric $\tilde{u}_{2 m}, \tilde{v}_{2 m-1}$, eigenfunctions must satisfy are, respectively,

System $S$

$$
\begin{gathered}
\{\mathscr{L}-2 M \bar{u}+\mathrm{i} \beta \operatorname{Re} \Omega\} \tilde{u}_{2 M-1}-\bar{u}^{\prime} \tilde{v}_{2 M-2}=0, \\
2(2 M-1)[\bar{u} \mathscr{D}+(\mathscr{D} \bar{u})] \tilde{u}_{2 M-1} \\
+\left\{\mathscr{M}+(2 M-1) \bar{u}^{\prime \prime}+\mathrm{i} \beta \operatorname{Re} \Omega\left[\mathscr{D}^{2}-\beta^{2}\right]\right\} \tilde{v}_{2 M-2}=0 ;
\end{gathered}
$$

System A

$$
\begin{gathered}
\left\{\mathscr{L}-(2 M+1) \bar{u}+\mathrm{i} \beta \operatorname{Re} \Omega \tilde{u}_{2 M}\right\} \tilde{u}_{2 M}-\bar{u}^{\prime} \tilde{v}_{2 M-1}=0, \\
4 M\{\bar{u} \mathscr{D}+(\mathscr{D} \bar{u})\} \tilde{u}_{2 M}+\left\{\mathscr{M}+2 M \bar{u}^{\prime \prime}+\mathrm{i} \beta \operatorname{Re} \Omega\left[\mathscr{D}^{2}-\beta^{2}\right]\right\} \tilde{v}_{2 M-1}=0 .
\end{gathered}
$$

Both systems $S$ and A may be collocated as either a temporal or a spatial eigenvalue problem and solved directly. While for most of the next section we follow the temporal concept, we close the presentation of the extended GH model by presenting spatial linear stability results pertinent to (4.40)-(4.41) and (4.42)-(4.43). First, though, the relationship of the one-dimensional extended GH model to the two-dimensional eigenvalue problem in which no assumptions on the chordwise dependence of the disturbance eigenfunctions are made is highlighted.

\section{Results of the extended Görtler-Hämmerlin model}

In view of the negligible cost of solving one-dimensional eigenvalue problems on present-day hardware, we have pursued three distinct paths in order to obtain the results to be presented below. First, all eigenvalues of systems (4.6)-(4.17) and (4.26)(4.34) were obtained by collocating and solving them directly using the QZ algorithm. Second, results delivered by the decoupled systems at the highest retained power of $x$, (4.21)-(4.24) and (4.35) (4.38), were monitored. Third, the eigenvalue problems (4.40)-(4.41) and (4.42)-(4.43) were solved. Finally, this series of solutions, obtained using Cray single-precision arithmetic was repeated on a workstation using IEEE double-precision arithmetic. In all cases identical eigenvalue problem results for the respective systems were obtained.

\subsection{Temporal linear instability in three dimensions, on the basis of the extended Görtler-Hämmerlin model}

Temporal eigenvalue results are obtained numerically by Chebyshev collocation of systems S and A. We monitor one Reynolds number value, $R e=800$, and two spanwise wavenumbers, $\beta=0.255$ and 0.3384631 , respectively corresponding to the zero-suction cases presented by Lin \& Malik (1996a) and Hall et al.(1984). We refrain from discussion of the numerics for the one-dimensional eigenvalue problem and refer the interested reader to Theofilis $(1998 a)$ for details. Results for the phase velocity $c_{\mathrm{r}}=\operatorname{Re}\{\Omega\} / \beta$ and growth rate $c_{\mathrm{i}}=\operatorname{Im}\{\Omega\} / \beta$ of the first four modes ordered 
$\mathrm{GH}(\mathrm{S} 1)$

(a) Resolution

\begin{tabular}{ccc} 
& & \\
\cline { 2 - 3 } Resolution & $c_{\mathrm{r}}$ & $c_{\mathrm{i}}\left(\times 10^{2}\right)$ \\
32 & 0.35877449 & 0.58317778 \\
48 & 0.35840978 & 0.58533042 \\
64 & 0.35840979 & 0.58532945 \\
80 & 0.35840979 & 0.58532945 \\
LM & 0.35840982 & 0.58532472
\end{tabular}

S2

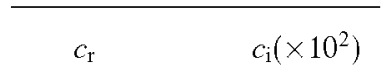

$\begin{array}{ccc}32 & 0.35777486 & 0.23178935 \\ 48 & 0.35743537 & 0.23430507 \\ 64 & 0.35743538 & 0.23430417 \\ 80 & 0.35743538 & 0.23430417 \\ \text { LM } & 0.35743540 & 0.23430006\end{array}$

GH (S1)

(b) Resolution

32
48
64
80

38

64

80
0.58532472
A1

\begin{tabular}{cl}
\hline$c_{\mathrm{r}}$ & $c_{\mathrm{i}}\left(\times 10^{2}\right)$ \\
0.35782552 & 0.40467537 \\
0.35791968 & 0.40989128 \\
0.35791968 & 0.40989108 \\
0.35791968 & 0.40989108 \\
0.35791970 & 0.40988667
\end{tabular}

A2

\begin{tabular}{cl}
\hline$c_{\mathrm{r}}$ & $c_{\mathrm{i}}\left(\times 10^{2}\right)$ \\
0.35687012 & 0.05375875 \\
0.35695685 & 0.05857525 \\
0.35695685 & 0.05857507 \\
0.35695685 & 0.05857507 \\
0.35695678 & 0.05857127
\end{tabular}

A1

\begin{tabular}{cl}
\hline$c_{\mathrm{r}}$ & $c_{\mathrm{i}}\left(\times 10^{2}\right)$ \\
0.37498493 & -0.11889215 \\
0.37514270 & -0.12683019 \\
0.37514273 & -0.12682731 \\
0.37514273 & -0.12682730
\end{tabular}

A2

\begin{tabular}{cc}
\hline$c_{\mathrm{r}}$ & $c_{\mathrm{i}}\left(\times 10^{2}\right)$ \\
0.37426270 & -0.37410119 \\
0.37441110 & -0.38102744 \\
0.37441113 & -0.38102525 \\
0.37441113 & -0.38102525
\end{tabular}

TABLE 2. Grid refinement history for the numerical solution of the one-dimensional extended GH model eigenvalue problems GH (S1), A1, S2 and A2 at $R e=800$ and $\beta=0.255(a)$ and $0.3384631(b)$. Also presented, denoted by LM, are the two-dimensional eigenvalue problem results of Lin \& Malik (1996a).

according to their physical significance, by reference to the growth rates, are presented in a grid sequencing form in table 2. For all modes presented, convergence of our results is demonstrated. Comparison with the results of Lin \& Malik (1996a) shows agreement up to the seventh to eighth decimal place. Discrepancies beyond this digit may be attributed to factors such as roundoff error in our iterative solution for the basic flow. We consider the excellent agreement of the results of the one-dimensional eigenvalue problem resulting from the extended GH model and the two-dimensional eigenvalue problem, which one has to solve if no assumption is made on the chordwise dependence of the two-dimensional linear disturbances, to be compelling evidence for us to claim that we have unravelled the structure underlying the two-dimensional solutions of Lin \& Malik (1996a). The two-dimensional eigenfunctions are separable 
solutions, amenable to analysis, in contrast to the statement to the contrary put forward by these authors. Consequently, in an incompressible swept attachment-line boundary layer in which the basic flow is taken to be the swept Hiemenz boundary layer there is no need for a two-dimensional eigenvalue problem to be solved. Results for the growth rate, frequency and spatial structure of three-dimensional disturbances can be recovered by solution of the one-dimensional eigenvalue problems resulting from of the structures (4.1) or (4.2), presented herein.

From a numerical point of view, the ability to recover linear instability results in three spatial dimensions by solution of an one-dimensional eigenvalue problem is of paramount significance. Eigenvalues and eigenfunctions pertinent to the model (4.40)(4.41) for symmetric or (4.42) (4.43) for antisymmetric modes results in a problem of approximately three orders of magnitude smaller size, taking between two and three orders of magnitude less time to solve (depending on the solution algorithm) than the eigenvalue problem (3.2)-(3.5). Specifically, solution to a one-dimensional eigenvalue problem is $O\left(\mathrm{~m}^{3}\right)$ faster and requires $O\left(\mathrm{~m}^{2}\right)$ less memory than that of the two-dimensional eigenvalue problem, in which $m$ points resolve the additional spatial direction. The precise savings may be inferred from the values of $m$ (which depend on the structure to be resolved) quoted in table 1. As such, the extended GH model presented herein is of interest for engineering applications aimed at transition prediction through linear mechanisms.

Even more significant, however, are the physical implications of the extended GH model for the analytical prediction of the spatial structure of the eigenfunctions in the chordwise direction. This result is itself amenable to asymptotic analysis; this is presented in $\$ 6$. Further, as has been mentioned by Lin \& Malik (1996a) also, the three-dimensional modes $\mathrm{A} 1, \mathrm{~S} 2, \mathrm{~A} 2, \ldots$ grow faster than mode $\mathrm{GH}$ in the chordwise direction, with the plausible implication that nonlinearity will be promoted by these higher polynomial modes if an arbitrary disturbance is projected upon this basis. The issue of subcritical instability of this flow may be associated, with turbulence being observed at $R e>245$, while linear theory (including the current model) predicts linear instability for $R e>583$. In order to verify and quantify the above postulate the extended GH model has been utilized to initialize threedimensional DNS at subcritical conditions; the results of this effort are presented in an accompanying paper (Theofilis \& Obrist 2003). In $\$ 5.3$ of the present work the closely related issue of convergence of the series expansions (4.1) and (4.2) in $x$ is addressed.

However, before turning to the latter issue and the asymptotic analysis, a further result of interest provided by the one-dimensional eigenfunctions pertaining to different modes in the extended GH model is discussed. We present in figure 4 the scaled real and imaginary parts of the linear eigenfunctions $\left(\tilde{u}_{1}, \tilde{v}_{0}\right),\left(\tilde{u}_{2}, \tilde{v}_{1}\right)$, $\left(\tilde{u}_{3}, \tilde{v}_{2}\right),\left(\tilde{u}_{4}, \tilde{v}_{3}\right)$ of modes $\mathrm{GH}, \mathrm{A} 1, \mathrm{~S} 2$, and $\mathrm{A} 2$, respectively. It can be clearly seen that all eigenfunctions monitored collapse into those of the $\mathrm{GH}$ mode. The implication of this result for experiments performed in the attachment-line boundary layer is clear. There exists in the flow a sequence of modes, obtained by alternatingly solving systems $\mathrm{S}$ and $\mathrm{A}$ at discrete values of $M$, which have frequencies lying very closely to one another and whose one-dimensional (in $y$ ) profile is identical. The features which may used to differentiate between the modes experimentally are either their different growth rates (with modes pertinent to larger $M$ being increasingly insignificant from a stability analysis point of view compared to those of low $M$ ) or their spatial structure in the chordwise direction; measurement of one-dimensional profiles in $y$ and subsequent scaling is inadequate. 

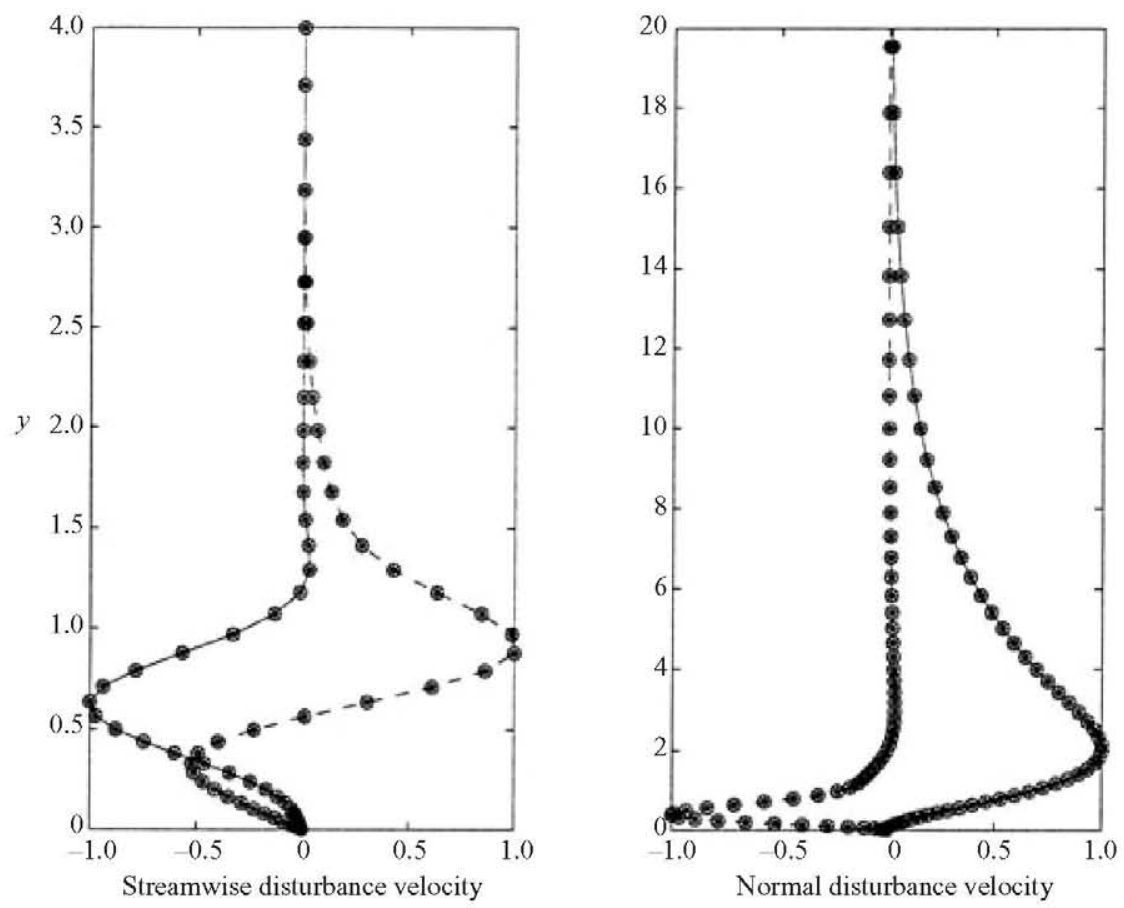

Figure 4. One-dimensional eigenfunctions $\tilde{u}$ and $\tilde{v}$. Solid line indicates the real and dashed the imaginary part of the eigenfunctions of the GH (S1) mode. Superimposed are the scaled eigenfunctions of mode A $1(0)$, mode S2 $(+)$ and mode A2 $(\times)$.

\subsection{Spatial linear instability in three dimensions}

So far we have discussed the results of the extended GH model within a temporal framework. It is straightforward to recast systems S and A as spatial linear stability problems, in which a real frequency parameter $\Omega$ is imposed and the response of the boundary layer is monitored in terms of the wavenumber $\operatorname{Re}\{\beta\}$ and growth rate $\operatorname{Im}\{\beta\}$ of the predicted eigenmode, after the discretized matrix eigenvalue problem has been solved for the determination of the complex eigenvalue $\beta$. The numerical complication arising when solving the spatial linear stability eigenvalue problem is that the eigenvalue appears nonlinearly in the governing equations. While a smaller problem is obtained by eliminating eigenfunctions from the system of equations resulting after linearization of the primitive form of the governing equations, the degree of nonlinearity of the eigenvalue increases. A numerical solution approach which is almost exclusively utilized for the one-dimensional linear spatial stability problem is that presented by Bridges \& Morris (1984). The method is based on augmenting the original problem with auxiliary unknowns accounting for the nonlinearity in the eigenvalue. This so-called 'companion matrix' approach has been successfully used for example, by Theofilis (1995) in the solution of the onedimensional spatial stability problem in the incompressible swept attachment-line boundary layer. Some implementational details of the companion matrix approach may be found in the Appendix.

While such an approach results in a discrete eigenproblem which is more expensive than that corresponding to a temporal analysis, it is well within current hardware capabilities. This is no longer true for BiGlobal instability analysis; for the solution 


$\begin{array}{cccccc}\text { GH (S1) } & \text { Resolution } & \beta_{\mathrm{r}} & \beta_{\mathrm{i}}\left(\times 10^{2}\right) & \beta_{\mathrm{r}} & \beta_{\mathrm{i}}\left(\times 10^{2}\right) \\ & 32 & 0.27704464 & -0.066868 & 0.34003905 & 0.058284 \\ & 48 & 0.27481340 & -0.226662 & 0.33846404 & 0.000283 \\ & 64 & 0.27481154 & -0.226961 & 0.33846355 & 0.000001 \\ & 80 & 0.27481154 & -0.226961 & 0.33846355 & 0.000000 \\ \mathrm{~A} 1 & 0.27481152 & -0.226959 & & \\ & & & & & \\ & \text { Resolution } & \beta_{\mathrm{r}} & \beta_{\mathrm{i}}\left(\times 10^{2}\right) & \beta_{\mathrm{r}} & \beta_{\mathrm{i}}\left(\times 10^{2}\right) \\ & 32 & 0.27502193 & -0.086292 & 0.33885284 & 0.134147 \\ & 48 & 0.27515280 & -0.105646 & 0.33864454 & 0.102767 \\ & 64 & 0.27515244 & -0.105987 & 0.33863756 & 0.102556 \\ \mathrm{~S} 2 & 80 & 0.27515245 & -0.105989 & 0.33863743 & 0.102555 \\ & \mathrm{~J} & 0.27515243 & -0.105988 & & \\ & \text { Resolution } & & & & \\ & 32 & 0.27769932 & 1.722107 & 0.34038577 & 0.265383 \\ & 48 & 0.27549093 & 0.151099 & 0.33880980 & 0.205326 \\ & 64 & 0.27548903 & 0.148146 & 0.33880924 & 0.205045 \\ & 80 & 0.27548905 & 0.148152 & 0.33880910 & 0.205053 \\ & \mathrm{~J} & 0.27548905 & 0.148157 & & \end{array}$

TABLE 3. Grid refinement history for the numerical solution of the spatial one-dimensional extended GH model eigenvalue problems GH (S1), A1 and S2 at $(a) R e=700$ and $\Omega=0.1017$ Joslin (1996b) and (b) $R e=800$ and $\Omega=0.1270977$ (Hall et al. 1984; Theofilis 1995). Also presented, denoted by $J$, the spatial partial-derivative eigenvalue problem result, as recovered in the spatial direct simulation of Joslin (1996b).

of the two-dimensional spatial linear eigenvalue problem it is imperative to use iterative algorithms. Consequently, the computing effort for a numerical solution of the spatial two-dimensional eigenvalue problem is at least as large as that of the temporal approach (Heeg \& Geurts 1998). Nevertheless, Joslin (1996b) has recovered the spatial two-dimensional linear eigenvalue problem results using his spatial direct numerical simulation code, albeit at a cost of approximately 13 CPU hours on a Cray C-90. Results of the spatial problem were provided by Joslin (1996b) with which our one-dimensional model, if accurately unravelling the flow physics, should compare.

We solved the spatial linear one-dimensional eigenvalue problems defined by systems $\mathrm{S}$ and $\mathrm{A}$ and present in table 3 the spatial eigenvalues $\beta$ pertaining to the case presented by Joslin, $R e=700, \Omega=0.1017$, as well as additional calculations performed at the conditions of Hall et al.(1984). In the first case the first two modes $\mathrm{GH}$ and $\mathrm{A} 1$ are unstable $(\operatorname{Im}\{\beta\}<0)$ while the third mode $\mathrm{S} 2$ is stable. In the second case the GH mode is neutrally stable while all other modes are stable. The comparison of the spatial linear stability results is as good as that in the temporal two-dimensional eigenproblem. The discrepancy between the results of Joslin (1996b) and the numerical solution of the one-dimensional spatial eigenvalue problem in which the extended GH model may be recast is less that one part in $10^{7}$, our approach taking typically a few seconds to deliver converged results on a workstation. Again, in addition to the fact that a one-dimensional eigenvalue problem describes the physics of instability in this flow at a negligible fraction of the computing effort of any approach previously 
presented, the significance of the agreement in the results of table 3 further underlines the ability of the extended GH model to reveal analytically the spatial structure of the modes responsible for linear instability in the incompressible swept attachment-line boundary layer.

\subsection{Convergence of the expansions (4.1) and (4.2)}

A final naturally arising question concerns realizability of the extended GH model in the chordwise direction, and the associated issue of the convergence properties of the series (4.1)-(4.2). This issue will be discussed further in the context of direct numerical simulations (Theofilis \& Obrist 2003), where physically plausible arguments are sought in order to select the chordwise extent of the integration domain, in lieu of an initialization of the simulations on a specific number of modes satisfying the extended GH model and their chordwise structure. Clearly, it may be argued that amplitude functions whose chordwise dependence is dominated by a polynomial function of $x$ cannot be expected to deliver realistic predictions as $x \rightarrow \infty$. While the higher the exponent in either series (4.1) or (4.2) the lower the growth (the stronger the damping) rate of the corresponding mode, the difference in damping rates at linearly subcritical Reynolds numbers may not be sufficient to compensate for the explosive growth of the series in $x$ away from the attachment line, a problem that would be aggravated if modes pertinent to large $M$-values are themselves amplified. Rather than resorting to arguments on nonlinearity moderating the growth in $x$, the properties of the coefficients $\left(\tilde{u}_{2 M-1}, \tilde{v}_{2 M-2}, \tilde{v}_{2 M-2}, \tilde{p}_{2 M-2}\right)^{\mathrm{T}}$ and $\left(\tilde{u}_{2 M}, \tilde{v}_{2 M-1}, \tilde{v}_{2 M-1}, \tilde{p}_{2 M-1}\right)^{\mathrm{T}}$ are examined next. These coefficients are recovered in a temporal framework by numerical solution of the coupled eigenvalue problems (4.6)-(4.17) and (4.26)-(4.34) which need to be solved if the amplitude functions of the extended GH model are sought.

Answering this question is interesting in a different context also. Joslin (1996a) has argued that the difference between the simulation results of Hall \& Malik (1986) and Jiménez et al. (1990) as regards two-dimensional nonlinear equilibria stems from the form of disturbance pressure assumed in the respective investigations, the former work having proposed that this quantity be taken constant, while in the latter work quadratic dependence of disturbance pressure on the chordwise coordinate was permitted. Joslin (1996a) went on to assert that in his three-dimensional simulations in the neighbourhood of the attachment line disturbance pressure could be approximated by a constant, which would support the form proposed by Hall \& Malik (1986); interestingly, a strong departure of the disturbance pressure from its near-independence of $x$ can be seen in the results of Joslin (1996a) at moderate (in terms of spanwise boundary-layer thickness) distances from the attachment line, a behaviour which might be attributable to the boundary conditions imposed at this end of the computational domain. In the course of the present investigation the properties of the coefficients in (4.1)-(4.2) reconcile the extended GH model with the numerical results of Joslin (1996a) and point to the resolution of this apparent dispute.

In order to compare results of the (linear) eigenvalue problems, physically relevant universal scales have been used. Disturbance-velocity amplitude functions of symmetric modes have been scaled using the $x$-independent maximum value of $\tilde{w}_{0}$, which on the one hand is the largest in magnitude amongst $\left(\tilde{u}_{1}, \tilde{v}_{0}, \tilde{w}_{0}\right)$ and on the other hand can be directly referred to the constant-thickness boundary layer along the spanwise direction. Disturbance velocity components of antisymmetric modes have been scaled on the only $x$-independent amplitude function pertinent to this family, $\tilde{u}_{0}$. The maximum of the GH disturbance pressure, $\tilde{p}_{0}$, is used as 

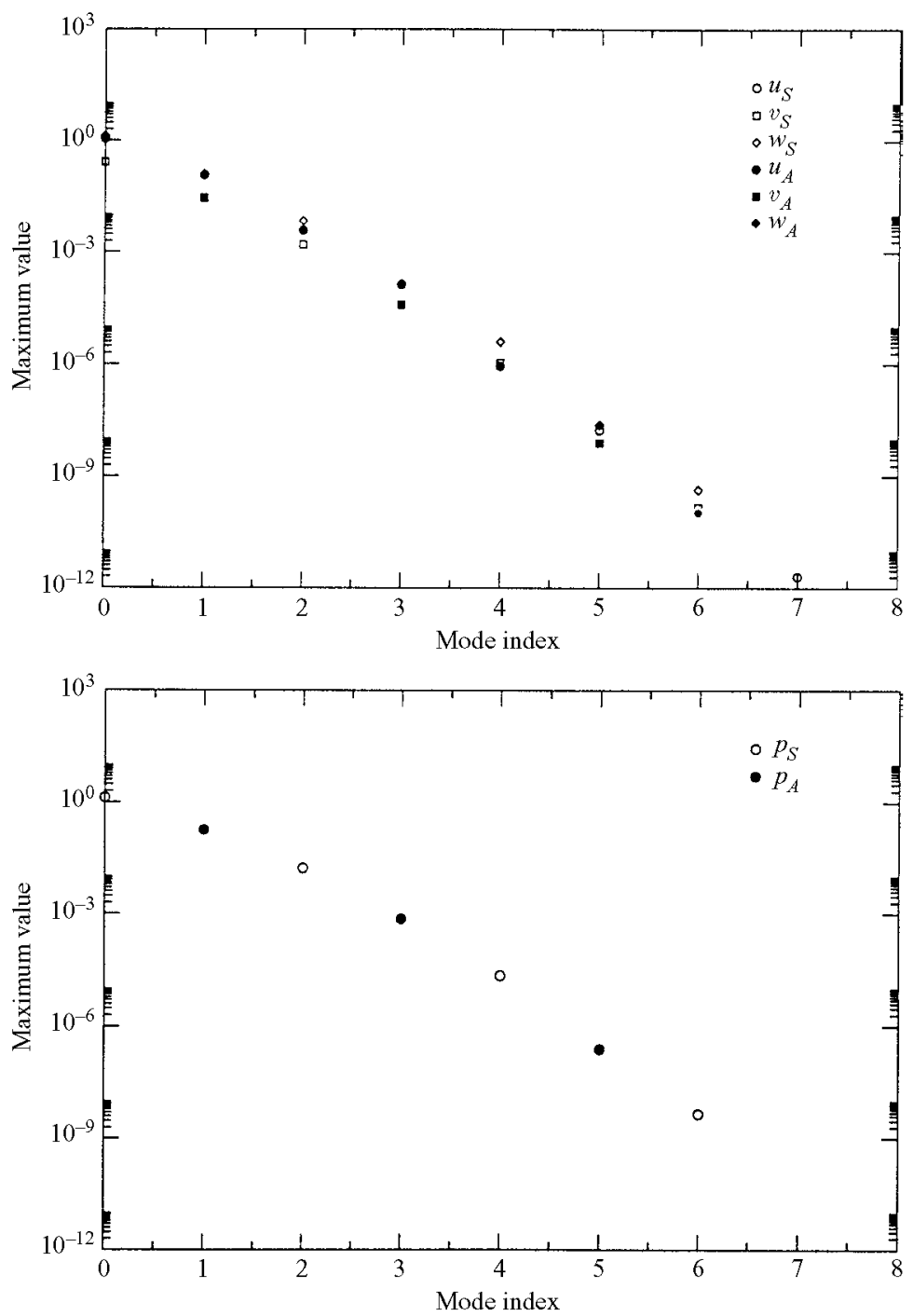

FIgURE 5. Maximum values of amplitude functions of the extended $\mathrm{GH}$ model against mode index $m$ at $R e=800, \beta=0.255 . u_{S}, v_{S}, w_{S}, p_{S}$ and $u_{A}, v_{A}, w_{A}, p_{A}$ denote maximum values of $\hat{u}_{m}, \hat{v}_{m}, \hat{w}_{m}, \hat{p}_{m}$ of symmetric and antisymmetric modes, respectively.

scale for the disturbance-pressure amplitude functions of all modes. Out of results obtained at several parameter-value combinations, only those pertaining to $(R e=$ $800, \beta=0.255)$ are presented. The eigenvalue problems for the recovery of the eigenfunctions $\hat{u}_{m}: m=0, \ldots, 7, \hat{v}_{m}: m=0, \ldots, 6, \hat{w}_{m}: m=0, \ldots, 6$, and $\hat{p}_{m}: m=$ $0, \ldots, 6$ have been solved, the respective maxima and $L_{2}$ norms of the (complex) coefficients have been calculated and the results are presented in figure 5; only results for the maxima of the amplitude functions are shown, those for the $L_{2}$ norm being qualitatively analogous. The significance of the expansion coefficients of the extended GH model at a given chordwise location is quantified in this figure. The exponential decay of the coefficients in the expansions (4.1)-(4.2) compensates for the polynomial growth of the respective amplitude function in $x$ at large distances from the leading 
edge and implies that the extended GH model delivers finite predictions away from the attachment line. The order of magnitude of each eigenmode at a given chordwise location near the attachment line can be inferred from the results of figure 5 as follows.

The $x$-independent coefficient $\tilde{w}_{0}$ pertaining to the GH mode and that of $\tilde{u}_{0}$ of mode A1 are taken at an amplitude such that linear theory holds; $;$ this amplitude value then scales the $y$-axis of the results of figure 5 . The coefficients of the disturbance-velocity components linearly growing in $x, \tilde{u}_{1}$ of the GH mode and $\tilde{v}_{1}, \tilde{w}_{1}$ of mode $\mathrm{A} 1$, are seen to be of $O\left(10^{-1}\right)$ smaller than the $x$-independent disturbance-velocity components, i.e. the former disturbances are expected to reach the level of the latter somewhere in the range $x \in[10,100]$. At the same distance normal to the leading edge the quadratically growing chordwise disturbance-velocity component of mode A1 is also expected to appear in the flow, since its amplitude function $\tilde{u}_{2}=O\left(10^{-2}\right)$. The same holds true for the spanwise and wall-normal disturbance velocity components (quadratically growing in $x$ ) of eigenmode $\mathrm{S} 2$ as well as for its cubically growing chordwise disturbance velocity component, the coefficient $\tilde{u}_{3}$ being of $O\left(10^{-3}\right)$; the argument can be carried forward to all higher modes, the amplitude functions pertinent to $x^{m}$ being more than an order of magnitude smaller than those at $x^{m-1}$. A corollary of this discussion is that by taking the amplitude of the (strongest) GH eigenmode to be consistent with the assumptions of linear theory, it is expected that a small number of the eigenmodes satisfying the extended GH model will be capable of describing the dynamics of the three-dimensional flow instability in an appreciable portion of the boundary layer in the neighbourhood of the attachment line.

Finally, turning to the issue of the form of disturbance pressure in three dimensions, figure 5 also provides the magnitude of the coefficients in an expansion of this in symmetric and antisymmetric parts. Disturbance pressure is seen to follow the same pattern as the disturbance-velocity components, the amplitude functions of a given family at a given order being approximately an order of magnitude smaller than those at the previous order (pertaining to the other family) and two orders of magnitude smaller than the previous member of the same family. On the basis of this result it can be argued that on the $(O, z, y)$-plane defined by the attachment line itself and the wall-normal, as well as very close to the attachment line, a near-constant disturbance pressure will prevail. This result is in line with that demonstrated in the three-dimensional DNS results of Joslin (1996a) and the assumption of Hall \& Malik (1986). On the other hand, the coefficient of the quadratic term in the disturbance-pressure expansion (4.20) is $O\left(10^{-2}\right)$ smaller than the $x$-independent pressure coefficient $\tilde{p}_{0}$, such that the term $\tilde{p}_{2} x^{2}$ will become comparable with $\tilde{p}_{0}$ at distances along the chord of the order of a few spanwise boundary layer thicknesses. Seen in this light, the form of disturbance pressure assumed by Jiménez et al. (1990) is more appropriate than that assumed by Hall \& Malik (1986) for the description of linear and nonlinear flow dynamics in all but the immediate neighbourhood of the attachment line. However, a linear disturbance-pressure component associated with mode $\mathrm{A} 1$ and, in turn, with an $x$-independent chordwise disturbance velocity component may also be present in the flow, alongside contributions from all highermode disturbances. In other words, neither of the forms of disturbance pressure assumed by Hall \& Malik (1986) nor Jiménez et al. (1990) is complete as far as

+ Strictly, the receptivity problem must be solved in order for the amplitudes of $\tilde{w}_{0}$ and $\tilde{u}_{0}$ to be provided. These amplitudes are, in principle, independent parameters of the problem. 
three-dimensional pressure perturbations are concerned, for the description of which one has to resort to either the partial-derivative eigenvalue problem or to the extended GH model.

\section{Asymptotic analysis for weakly non-parallel flow}

We discuss here an alternative approach to arrive at the results of $\S 5$, which also offers a simplification of the attachment-line stability analysis compared with solution of the partial-derivative eigenvalue problem or DNS, using the multiple-scales method developed for weakly non-parallel boundary layers, which also arrives at the extended GH model description of three-dimensional instability of the flow in question. The advantage of the asymptotic analysis is that it offers quantification, in terms of a Reynolds-number-dependent small parameter, of the error of a given approximation. Details of this method can be found, e.g. in Nayfeh (1980) and Zhigulev \& Tumin (1987).

A solution of the incompressible continuity and Navier-Stokes equations $Q(x, y, z, t)$ is decomposed as before using (3.1) with $Q_{b}=(x \bar{U}, \bar{V}, \bar{W}, \bar{P})^{\mathrm{T}}$ indicating basic-flow velocity and pressure components and $Q_{p}=(\hat{u}, \hat{v}, \hat{w}, \hat{p})^{\mathrm{T}}$ denoting perturbations to the basic flow. If $\Omega$ is a real parameter, the complex eigenvalue $\beta\left(\Omega, R e, x_{1}\right)$ is associated with the spatial instability problem. If $\beta$ is real parameter, the complex eigenvalue $\Omega\left(\beta, R e, x_{1}\right)$ is relevant to the temporal instability problem. The spatial stability analysis is presented first.

\subsection{Asymptotic analysis of the spatial eigenvalue problem}

Assuming that the Reynolds number is large, a small parameter $\epsilon=1 /$ Re and slow variables $x_{1}=\epsilon x, z_{1}=\epsilon z$ are introduced. In the framework of the multiple-scales approach, the disturbance amplitude vector is expressed as

$$
\boldsymbol{Q}_{p}=\boldsymbol{Q}_{0}\left(x_{1}, z_{1}, y\right)+\epsilon \boldsymbol{Q}_{1}\left(x_{1}, z_{1}, y\right)+\cdots .
$$

Substituting (3.1) and (6.1) into the linearized Navier-Stokes equations, the eigenvalue problem of the first-order approximation

$$
\begin{gathered}
\mathscr{B} \hat{u}_{0}+x_{1} \bar{U}^{\prime} \hat{v}_{0}=\frac{1}{R e}\left(\hat{u}_{0}^{\prime \prime}-\beta^{2} \hat{u}_{0}\right), \\
\mathscr{B} \hat{v}_{0}+\hat{p}_{0}^{\prime}=\frac{1}{R e}\left(\hat{v}_{0}^{\prime \prime}-\beta^{2} \hat{v}_{0}\right), \\
\mathscr{B} \hat{w}_{0}+\bar{W}^{\prime} \hat{v}_{0}+\mathrm{i} \beta \hat{p}_{0}=\frac{1}{R e}\left(\hat{w}_{0}^{\prime \prime}-\beta^{2} \hat{w}_{0}\right), \\
\hat{v}_{0}^{\prime}+\mathrm{i} \beta \hat{w}_{0}=0,
\end{gathered}
$$

together with

$$
\hat{u}_{0}=\hat{v}_{0}=\hat{w}_{0} \quad \text { at } \quad y=0 \quad \text { and } \quad y \rightarrow \infty,
$$

is obtained, with $\mathscr{B}=\mathrm{i}(\beta \bar{W}-\Omega)$. In this system of equations higher-order viscous terms are retained in order for a standard Orr-Sommerfeld-like operator for threedimensional boundary-layer flow to be obtained. This permits eigensolutions to be recovered which are uniformly valid across the boundary layer. The problem (6.2)(6.6) contains $x_{1}$ as a parameter; it can be written in the compact form

$$
\begin{gathered}
\mathscr{P}\left(\Omega, \beta, R e, x_{1}\right) \boldsymbol{Q}_{0}=0, \\
\boldsymbol{Q}_{01}=\boldsymbol{Q}_{02}=\boldsymbol{Q}_{03}=0 \quad \text { at } \quad y=0 \text { and } y \rightarrow \infty,
\end{gathered}
$$


where $\mathscr{P}$ is a matrix differential operator. A solution of the homogeneous problem (6.2) (6.6) can be expressed as

$$
\boldsymbol{Q}_{0}=C\left(x_{1}, z_{1}\right)\left(x_{1} \hat{u}_{01}(y), \hat{v}_{00}(y), \hat{w}_{00}(y), \hat{p}_{00}(y)\right)^{\mathrm{T}} .
$$

Substituting (6.8) into equations (6.2)-(6.5) a problem which does not depend on $x_{1}$ is obtained:

$$
\begin{gathered}
\mathscr{B} \hat{u}_{01}+\bar{U}^{\prime} \hat{v}_{00}=\frac{1}{R e}\left(\hat{u}_{01}^{\prime \prime}-\beta^{2} \hat{u}_{01}\right), \\
\mathscr{B} \hat{v}_{00}+\hat{p}_{00}^{\prime}=\frac{1}{R e}\left(\hat{v}_{00}^{\prime \prime}-\beta^{2} \hat{v}_{00}\right), \\
\mathscr{B} \hat{w}_{00}+\bar{W}^{\prime} \hat{v}_{00}+\mathrm{i} \beta \hat{p}_{00}=\frac{1}{R e}\left(\hat{w}_{00}^{\prime \prime}-\beta^{2} \hat{w}_{00}\right), \\
\hat{v}_{00}^{\prime}+\mathrm{i} \beta \hat{w}_{00}=0,
\end{gathered}
$$

subject to

$$
\hat{u}_{10}=\hat{v}_{00}=\hat{w}_{00} \quad \text { at } \quad y=0 \text { and } \quad y \rightarrow \infty .
$$

Solution of this problem delivers the eigenvalue $\beta=\beta_{0}(\Omega, R e)$ of the first-order approximation. Note that equation (6.9) is decoupled from equations (6.10)-(6.12). The latter can be transformed to the Orr-Sommerfeld equation for a two-dimensional parallel boundary layer with the velocity profile $\bar{W}(y)$.

In the second-order approximation the disturbance vector is expressed in the form

$Q_{1}$

$$
=C\left(x_{1}, z_{1}\right)\left(x_{1} \hat{u}_{11}\left(x_{1}, z_{1}, y\right)+\hat{u}_{10}\left(x_{1}, z_{1}, y\right), \hat{v}_{10}\left(x_{1}, z_{1}, y\right), \hat{w}_{10}\left(x_{1}, z_{1}, y\right), \hat{p}_{10}\left(x_{1}, z_{1}, y\right)\right)^{\mathrm{T}} .
$$

The vector $\boldsymbol{Q}_{10}=\left(\hat{u}_{11}, \hat{v}_{10}, \hat{w}_{10}, \hat{p}_{10}\right)^{\mathrm{T}}$ is a solution of the inhomogeneous problem

$$
\begin{gathered}
\mathscr{P}_{0} \boldsymbol{Q}_{10}+\frac{x_{1}}{C} \frac{\partial C}{\partial x_{1}} \boldsymbol{G}_{1}(y)+\frac{1}{C} \frac{\partial C}{\partial z_{1}} \boldsymbol{G}_{2}(y)+\boldsymbol{G}_{3}(y)=0, \\
\hat{u}_{11}=\hat{v}_{10}=\hat{w}_{10} \quad \text { at } \quad y=0 \quad \text { and } \quad y \rightarrow \infty .
\end{gathered}
$$

Here $\mathscr{P}_{0}$ is the linear operator of the homogeneous system (6.9)-(6.13); the vectors $G_{j}(y)$ are expressed in the explicit form

$$
\begin{aligned}
& \boldsymbol{G}_{1}=\left(\bar{U} \hat{u}_{01}, \bar{U} \hat{v}_{00}, \bar{U} \hat{w}_{00}, \hat{u}_{01}\right)^{\mathrm{T}}, \\
& \boldsymbol{G}_{2}=\left(\bar{W} \hat{u}_{01}, \bar{W} \hat{v}_{00}, \bar{W} \hat{w}_{00}+\hat{p}_{00}, \hat{w}_{00}\right)^{\mathrm{T}}, \\
& \boldsymbol{G}_{3}=\left(2 \bar{U} \hat{u}_{01}+\bar{V} \hat{u}_{01}^{\prime},\left(\bar{V} \hat{v}_{00}\right)^{\prime}, \bar{V} \hat{w}_{00}^{\prime}, \hat{u}_{01}\right)^{\mathrm{T}}
\end{aligned}
$$

The component $\hat{u}_{10}$ is a solution of the inhomogeneous problem

$$
\begin{gathered}
\mathrm{i}\left(\beta_{0} \bar{W}-\Omega\right) \hat{u}_{10}-\frac{1}{R e}\left(\hat{u}_{10}^{\prime \prime}-\beta_{0}^{2} \hat{u}_{10}\right)+\frac{1}{C} \frac{\partial C}{\partial x_{1}} \hat{p}_{00}=0, \\
\hat{u}_{10}=0 \quad \text { at } y=0 \text { and } y \rightarrow \infty .
\end{gathered}
$$

The problem (6.15)-(6.16) has a non-trivial solution if the inhomogeneous part of equation (6.15) is orthogonal to the correspondent solution $\boldsymbol{Z}$ of the adjoint problem. This solvability condition provides the partial-differential equation for the amplitude 
function $C\left(x_{1}, z_{1}\right)$ :

$$
\left\langle\boldsymbol{G}_{1}, \boldsymbol{Z}\right\rangle x_{1} \frac{\partial \boldsymbol{C}}{\partial x_{1}}+\left\langle\boldsymbol{G}_{2}, \boldsymbol{Z}\right\rangle \frac{\partial \boldsymbol{C}}{\partial z_{1}}+\left\langle\boldsymbol{G}_{3}, \boldsymbol{Z}\right\rangle \boldsymbol{C}=0,
$$

where the scalar product is defined as

$$
\langle\boldsymbol{G}, \boldsymbol{Z}\rangle=\int_{0}^{\infty} \sum_{j=1}^{4} G_{j} Z_{j} \mathrm{~d} y .
$$

Equation (6.23) has the following set of partial solutions:

$$
\begin{gathered}
C=x_{1}^{n} \exp \left\{\mathrm{i} \beta_{1} \zeta_{1}\right\}, \\
\beta_{1}(n)=\mathrm{i} \frac{n\left\langle\boldsymbol{G}_{1}, \boldsymbol{Z}\right\rangle+\left\langle\boldsymbol{G}_{3}, \boldsymbol{Z}\right\rangle}{\left\langle\boldsymbol{G}_{2}, \boldsymbol{Z}\right\rangle} .
\end{gathered}
$$

For $n=0$ the last term in equation (6.21) is zero and the correspondent homogeneous problem (6.21)-(6.22) has the trivial solution $\hat{u}_{10}=0$. In this case, the disturbance vector of the first-order approximation is expressed as

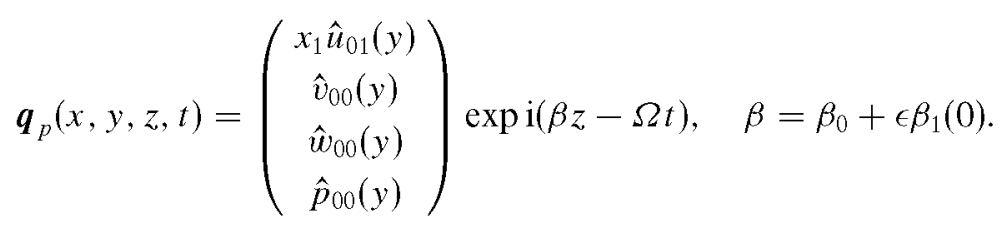

For $n \neq 0$, a solution of equation (6.21) is presented in the form $\hat{u}_{10}=x_{1}^{-1} \tilde{u}_{10}(y)$ where $\tilde{u}_{10}$ is obtained from solving the inhomogeneous problem

$$
\begin{aligned}
\mathrm{i}\left(\beta_{0} \bar{W}-\Omega\right) \tilde{u}_{10}-\frac{1}{R e}\left(\tilde{u}_{10}^{\prime \prime}-\beta_{0}^{2} \tilde{u}_{10}\right)+n \hat{p}_{00} & =0, \\
\tilde{u}_{10}=0 \quad \text { at } y=0 \text { and } y & \rightarrow \infty .
\end{aligned}
$$

In this case, the disturbance vector is expressed as

$$
\boldsymbol{q}_{p}(x, y, z, t)=x_{1}^{n}\left(\begin{array}{c}
x_{1} \hat{u}_{01}(y)+x_{1}^{-1} \tilde{\boldsymbol{u}}_{10} \\
\hat{v}_{00}(y) \\
\hat{w}_{00}(y) \\
\hat{p}_{00}(y)
\end{array}\right) \exp \mathrm{i}(\beta z-\Omega t), \quad \beta=\beta_{0}+\epsilon \beta_{1}(n) .
$$

Since the disturbance amplitude is finite on the attachment line, $x_{1}=0$, the solution (6.29) is valid for $n \geqslant 1$. Taking discrete values of the integer parameter $n$ one obtains a family of attachment-line boundary layer modes. Solutions with $n=0,2, \ldots$ correspond to symmetric modes S1, S2, ., and solutions with $n=1,3, \ldots$ correspond to antisymmetric modes $\mathrm{A} 1, \mathrm{~A} 2, \ldots$

An analogous analysis can be performed for the temporal stability problem. In this case, the slow variables $t_{1}=\epsilon t$ and $x_{1}=\epsilon x$ are introduced and the disturbance amplitude is represented as

$$
\boldsymbol{Q}_{p}=\boldsymbol{Q}_{0}\left(x_{1}, t_{1}, y\right)+\epsilon \boldsymbol{Q}_{1}\left(x_{1}, t_{1}, y\right)+\cdots .
$$

In the first-order approximation, the disturbance vector is expressed in the form

$$
\boldsymbol{q}_{p}(x, y, z, t)=x_{1}^{n}\left(\begin{array}{c}
x_{1} \hat{\boldsymbol{u}}_{01}(y) \\
\hat{v}_{00}(y) \\
\hat{w}_{00}(y) \\
\hat{p}_{00}(y)
\end{array}\right) \exp \mathrm{i}(\beta z-\Omega t)
$$




$$
\begin{gathered}
\Omega(n, \beta, R e)=\Omega_{0}(\beta, R e)+\epsilon \Omega_{1}(n, \beta, R e)+O\left(\epsilon^{2}\right), \\
\Omega_{1}(n, \beta, R e)=-\mathrm{i} \frac{n\left\langle\boldsymbol{G}_{1}, \boldsymbol{Z}\right\rangle+\left\langle\boldsymbol{G}_{3}, \boldsymbol{Z}\right\rangle}{\left\langle\boldsymbol{G}_{4}, \boldsymbol{Z}\right\rangle}
\end{gathered}
$$

where $\boldsymbol{G}_{4}=\left(\hat{u}_{01}, \hat{v}_{00}, \hat{w}_{00}, 0\right)^{T}$.

\subsection{Connection with the analysis of $\$ 4$}

One can compare the asymptotic solutions (6.29) with the solutions of $\S 4$ expressed in the form of truncated series. As an example, the first symmetric mode with the amplitude vector

$$
Q_{p}=\left(x \tilde{u}_{1}(y), \tilde{v}_{0}(y), \tilde{w}_{0}(y), \tilde{p}_{0}(y)\right)^{T}
$$

is considered. Components of this vector are expanded with respect to $\epsilon$ as

$$
\begin{aligned}
\tilde{u}_{1} & =\epsilon \tilde{u}_{10}+\epsilon^{2} \tilde{u}_{11}+\cdots, \\
\tilde{v}_{0} & =\tilde{v}_{00}+\epsilon \tilde{v}_{01}+\cdots, \\
\tilde{w}_{0} & =\tilde{w}_{00}+\epsilon \tilde{w}_{01}+\cdots, \\
\tilde{p}_{0} & =\tilde{p}_{00}+\epsilon \tilde{p}_{01}+\cdots, \\
\beta & =\beta_{0}+\epsilon \beta_{1}+\cdots .
\end{aligned}
$$

Substituting these expansions into the system (4.6)-(4.9) and keeping the first-order terms, a problem which is equivalent to (6.9)-(6.13) resulting from the present asymptotic analysis is obtained. In the second-order approximation, the inhomogeneous system of equations

$$
\mathscr{P}_{0}\left(\begin{array}{c}
\tilde{u}_{11} \\
\tilde{v}_{01} \\
\tilde{w}_{01} \\
\tilde{p}_{01}
\end{array}\right)+\left(\begin{array}{c}
\left(\mathrm{i} \beta_{1} \bar{W}+2 \bar{U}\right) \tilde{u}_{10}+\bar{V} \tilde{u}_{10}^{\prime} \\
\mathrm{i} \beta_{1} \bar{W} \tilde{v}_{00}+\left(\bar{V} \tilde{v}_{00}\right)^{\prime} \\
\mathrm{i} \beta_{1}\left(\bar{W} \tilde{w}_{00}+\tilde{p}_{00}\right)+\bar{V} \tilde{w}_{00}^{\prime} \\
\mathrm{i} \beta_{1} \tilde{w}_{00}+\tilde{u}_{10}
\end{array}\right)=0
$$

is obtained, which is identical with equations (6.15). Thus, the asymptotic solution (6.27) approximates the eigenfunction with accuracy $O(\epsilon)$ and the eigenvalue with accuracy $O\left(\epsilon^{2}\right)$. An analogous comparison can be made for the symmetric modes $\mathrm{S} 2, \mathrm{~S} 3, \ldots$ taking $n=2,4, \ldots$ and the antisymmetric modes $\mathrm{A} 1, \mathrm{~A} 2, \ldots$ taking $n=$ $1,3, \ldots$

\subsection{An algorithm for the calculation of eigenvalues of three-dimensional disturbances with $O\left(\epsilon^{2}\right)$ accuracy}

Summarizing the asymptotic analysis of linear instabilities in the three-dimensional attachment-line boundary layer the following algorithm for the calculation of the eigenvalues

$$
\beta(n, \operatorname{Re}, \Omega)=\beta_{0}(\operatorname{Re}, \Omega)+\epsilon \beta_{1}(n, \operatorname{Re}, \Omega)
$$

with $O\left(\epsilon^{2}\right)$ accuracy is formulated:

Solve the Orr-Sommerfeld-like problem (6.9)-(6.13).

Solve the corresponding adjoint problem and obtain the eigenvalue $\beta_{0}(R e, \Omega)$ of the first-order approximation.

Calculate the eigenvalues $\beta(n, R e, \Omega)$ of symmetric eigenmodes from (6.41) using the analytical expression (6.26) and $n=0,2, \ldots$ to calculate $\beta_{1}$. 


\begin{tabular}{rccrccc} 
& \multicolumn{2}{c}{ system $(3.7)$} & & \multicolumn{2}{c}{ system $(6.31)-(6.33)$} \\
\cline { 2 - 3 } \cline { 6 - 7 } Mode & $c_{\mathrm{r}}$ & $c_{\mathrm{i}}\left(\times 10^{2}\right)$ & & $n$ & $c_{\mathrm{r}}$ & $c_{\mathrm{i}}\left(\times 10^{2}\right)$ \\
GH (S1) & 0.358410 & 0.585325 & & 0 & 0.358469 & 0.584831 \\
A1 & 0.357920 & 0.409887 & 1 & 0.358002 & 0.410754 \\
S2 & 0.357435 & 0.234300 & 2 & 0.357534 & 0.236677 \\
A2 & 0.356957 & 0.058571 & 3 & 0.357067 & 0.062600
\end{tabular}

TABLE 4. Comparison of temporal eigenvalues obtained by asymptotic analysis at $O\left(\epsilon^{2}\right)$ against two-dimensional eigenvalue problem results at $\kappa=0, \operatorname{Re}=800, \beta=0.255$.

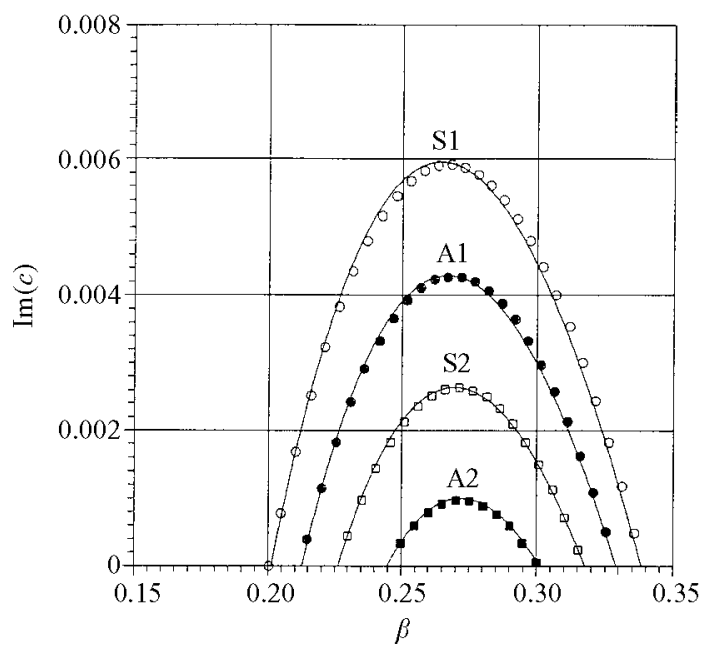

FIGURE 6. Distributions of $c_{\mathrm{i}}(\beta)$ for symmetric $(\mathrm{GH} / \mathrm{S} 1, \mathrm{~S} 2)$ and antisymmetric $(\mathrm{A} 1, \mathrm{~A} 2)$ modes at $R e=800$; solid lines: asymptotic analysis, symbols: partial-derivative eigenvalue problem solution.

Calculate the eigenvalues $\beta(n, R e, \Omega)$ of antisymmetric eigenmodes also from (6.41) using the analytical expression (6.26) and $n=1,3, \ldots$ to calculate $\beta_{1}$.

In a manner analogous with numerical solution of the ordinary-differential-equation eigenvalue problems (4.40)-(4.43) this algorithm permits orders-of-magnitude faster calculations of the attachment-line stability characteristics when compared with either solution of the spatial or temporal partial-derivative eigenvalue problems or spatial DNS. In table 4 results of the algorithm for the complex phase velocity $c=c_{\mathrm{r}}+\mathbf{i} c_{\mathrm{i}}=$ $\Omega(\beta, R e) / \beta$ are compared to those of the temporal partial-derivative eigenvalue problem for modes $\mathrm{S} 1, \mathrm{~A} 1, \mathrm{~S} 2$ and $\mathrm{A} 2$ at $R e=800, \beta=0.255$. The relative error between the asymptotic analysis results and the exact solutions of the partial-derivative eigenvalue problem is $O(0.01 \%)$ for the most unstable eigenmode and increases to a few percent for the less-amplified higher modes. The distribution of $c_{\mathrm{i}}(\beta)$ at $R e=800$ is shown in figure 6. Stability characteristics predicted by the multiple-scales method (solid lines) are very close to those of solution of the partial-derivative eigenvalue problem (symbols). The asymptotic analysis calculations indicate that both terms in equation (6.33) have negative imaginary part. Consequently the most unstable 


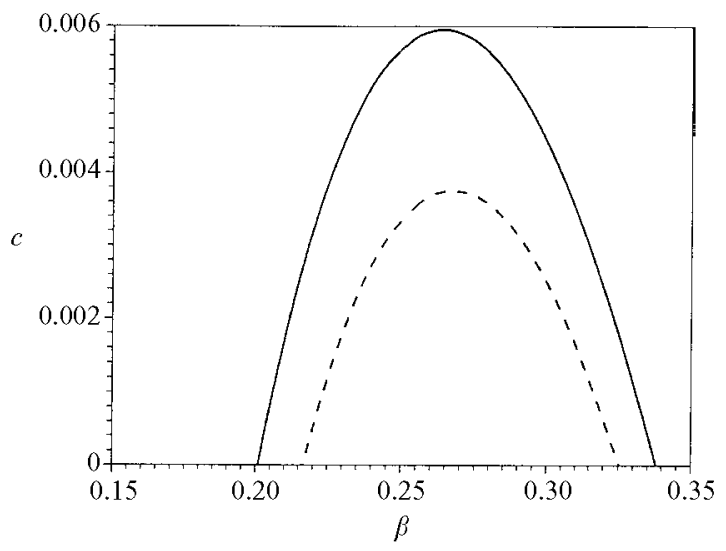

FIguRE 7. Distribution of $c_{\mathrm{i}}(\beta)$ for the first symmetric mode GH (S1) at $R e=800$; dashed line: the first-order approximation, solid line: the second-order approximation.

eigenmode is $\mathrm{S} 1$, and the growth rates follow the inequality $\Omega_{\mathrm{i}, \mathrm{S} 1}>\Omega_{\mathrm{i}, \mathrm{A} 1}>\Omega_{\mathrm{i}, \mathrm{S} 2}>$ $\Omega_{\mathrm{i}, \mathrm{A} 2}>\cdots$, a conclusion consistent with the results of numerical solution of the partial-derivative eigenvalue problem. Figure 7 illustrates the non-parallel effect on the growth rate of mode $\mathrm{S} 1$; the difference between the first-order approximation (dashed line) and the second-order approximation (solid line) is more than $40 \%$, i.e. the non-parallel correction $\beta_{1}(n)$ should be accounted for in order for the prediction of the disturbance amplification to be improved.

Equation (6.31) shows that the eigenfunctions $\hat{u}_{01}(y), \hat{v}_{00}(y), \hat{w}_{00}(y)$, and $\hat{p}_{00}(y)$ are identical for both symmetric and antisymmetric modes with accuracy $O(1 / R e)$. In figure 8 the function $w(y)=\left|\hat{w}_{00}(y)\right|$ (solid line), normalized such that $\max \{w\}=1$, is compared with the numerical result of the partial-derivative eigenvalue problem for the S1 and A1 eigenmodes (symbols); the analogous comparison for the S2 and A2 eigenmodes is also shown in figure 8. As already stated, the approximation of the eigenfunctions provided by the asymptotic analysis is within $O(\epsilon)$ of the exact numerical result, and both sets of eigenfunctions can be seen to be approximated well by the asymptotic analysis. In other words, with accuracy $O(1 / R e)$, the eigenfunctions of both symmetric and antisymmetric modes have a universal structure given by equation (6.31). If the S1 eigenfunction is known, all others can be reproduced using the multiplication factor $x_{1}^{n}$. This result too is in line with the numerical prediction of the extended GH model eigenvalue problem shown in figure 4. In summary, the asymptotic analysis based on the multiple-scales method provides a very effective and robust algorithm for stability analysis of the attachment-line boundary layer, as an alternative to the extended GH model discussed in $\S 4$, and delivers results consistent with those of this model and, in addition, offers an estimate of the order of magnitude of the accuracy of the approximation made in terms of the inverse-Reynolds-number small parameter.

\section{Direct numerical simulation}

\subsection{Numerical method}

The analytical results presented in the previous sections are next verified by threedimensional DNS using a full nonlinear code to monitor the linear development of the 

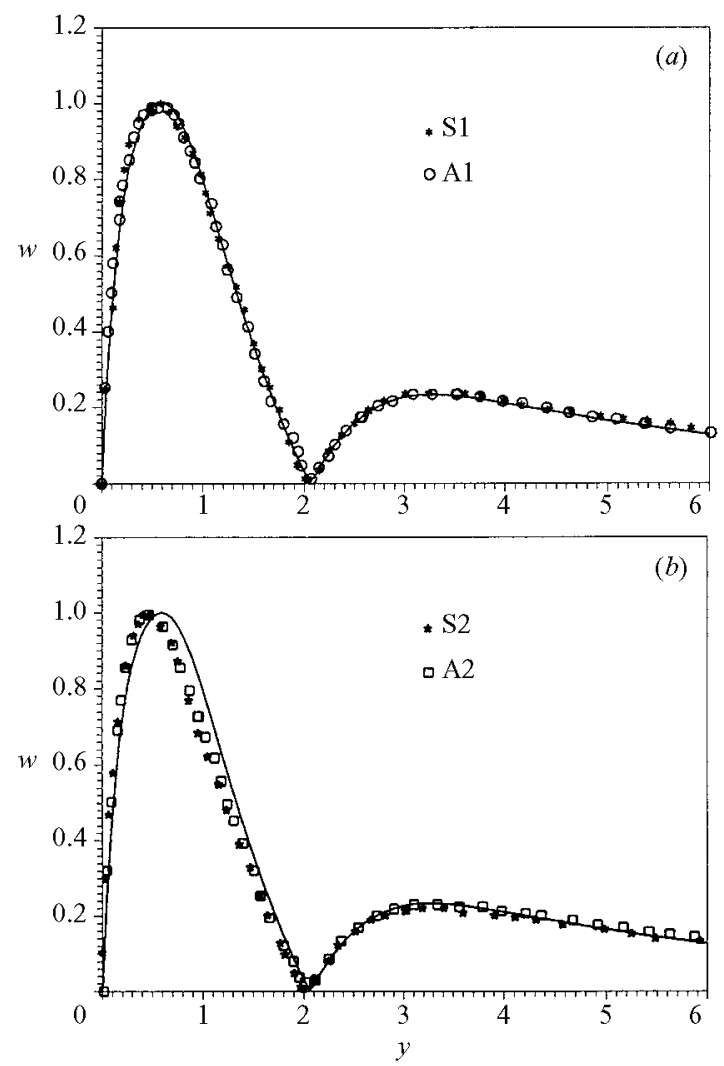

FIGURE 8. (a) Normalized eigenfunction $w(y)$ for the first symmetric $(\mathrm{GH} / \mathrm{S} 1)$ and antisymmetric (A1) modes. (b) Normalized eigenfunction $w(y)$ for the second symmetric (S2) and antisymmetric (A2) modes. $R e=800, \beta=0.255$; solid lines: asymptotic analysis, symbols: partial-derivative eigenvalue problem solution.

three-dimensional extended GH model disturbances. The incompressible continuity and Navier-Stokes equations are stated in a velocity-vorticity formulation. The DNS code is an extension of that of Lundbladh et al. (1994) and uses spectral methods to discretize the spatial operator, in particular a Fourier expansion in the chord and spanwise directions, $x$ and $z$ respectively, and Chebyshev polynomials in the wall-normal direction $y$. There is a mixed implicit-explicit time integration method: a Crank-Nicolson scheme is used for the viscous terms and a third-order RungeKutta scheme for the nonlinear terms. In the wall-normal direction the Chebyshev transformation is done on a Gauss-Lobatto grid. To account for the semi-infinite physical domain the Gauss-Lobatto points are stretched to a finite interval $y \in$ $\left[0, L_{y}\right]$ by an algebraic transformation which clusters half of the collocation points between the wall and an appropriately chosen location $y_{1 / 2}$ inside the boundary layer (Canuto et al. 1987); in the scalings of $\S 2, y_{1 / 2}=4$ and $L_{y} \geqslant 150$ have been used throughout the following simulations. The two horizontal directions are considered homogeneous and treated as periodic, an approach which is in line with the flow physics only as far as the spanwise direction is concerned. In order to obtain periodicity in the chordwise direction we use the fringe-region technique (Spalart 1988). 


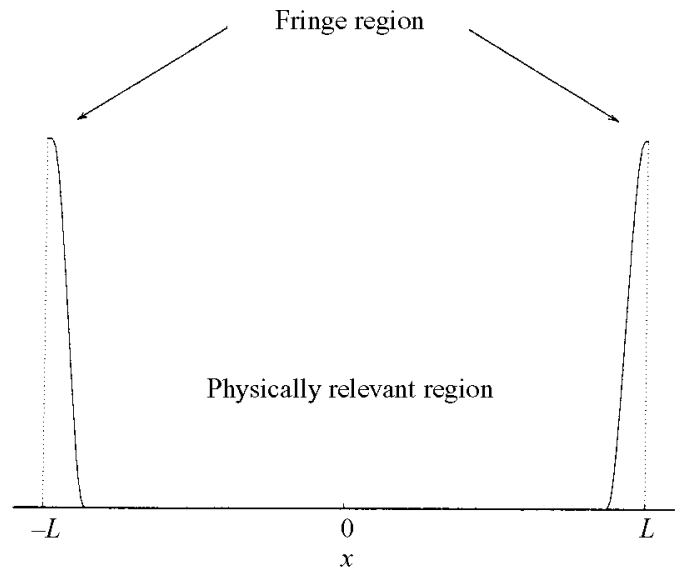

FIGURE 9. Schematic representation of the fringe function $\lambda(x)$ (Obrist 2000).

\subsection{The fringe-region technique}

The fringe-region treatment of the chordwise direction, schematically depicted in figure 9, consists of adding a linear damping term to the governing equation

$$
\frac{\partial}{\partial t} u+\mathscr{L}_{s}(u)=\lambda(x)[U(x, t)-u],
$$

where $u$ is the dependent variable, $\mathscr{L}_{s}$ is the - typically elliptic - spatial operator, and $\lambda(x)$ is a so-called 'fringe function'. The inhomogeneity $U(x, t)$ may be arbitrarily chosen and can act as a forcing for an inflow boundary condition - as used for example in spatial DNS of a channel flow configuration. In the DNS of a leadingedge boundary layer the fringe forcing does not need to generate any inflow boundary condition. In this case the inhomogeneity $U(x, t)$ is chosen to be identically zero.

The fringe function $\lambda(x)$ is the main design parameter for the fringe-region technique. It is zero everywhere except inside an ideally short interval called the 'fringe region'. A heuristic interpretation of the fringe-region technique begins with the assumption that $\lambda(x)$ is so large inside the fringe region that the contribution from the the spatial operator $\mathscr{L}_{s}$ can be neglected. This permits rewriting (7.1) as

$$
\frac{\partial}{\partial t} u \approx \begin{cases}-\mathscr{L}_{s}(u) & \text { outside the fringe region, } \\ \|\lambda\|[U-u] & \text { inside the fringe region. }\end{cases}
$$

This suggests that the governing equation $u_{t}+\mathscr{L}_{s}(u)=0$ is simulated outside the fringe region, whereas a linear ODE is solved inside the fringe region, such that $u$ is forced towards $U$.

A smooth fringe function $\lambda(x)$ is chosen in order to minimize the influence of the fringe region on the physically relevant part of the simulation domain, since a too steep slope would cause strong reflection of waves. In the present code

$$
\begin{aligned}
& \lambda(x)=\hat{\lambda}\left[S\left(\frac{x-x_{\text {start }}}{d_{\text {rise }}}\right)-S\left(\frac{x-x_{\text {end }}}{d_{\text {fall }}}+1\right)\right], \\
& S(x)= \begin{cases}0, & x \leqslant 0 \\
1 /\left[1+\exp \left(\frac{1}{x-1}+\frac{1}{x}\right)\right], & 0<x<1 \\
1, & x \geqslant 1\end{cases}
\end{aligned}
$$


is set. The parameter $\hat{\lambda}$ defines the magnitude of the fringe function, whereas the parameters $x_{\text {start }}, x_{\text {end }}, d_{\text {rise }}$, and $d_{\text {fall }}$ define the shape of the fringe function via the smooth step function $S(x)$. In contrast to the buffer-domain technique of Spalart (1988) the present version of the fringe forcing is linear; it was first used by Lundbladh et al. (1994) to simulate spatially evolving disturbances in a channel flow. Validation calculations show that the fringe forcing contaminates the physically relevant region upstream. Therefore the fringe region has to be positioned far enough from the attachment line, in a region where the basic flow has a more parabolic character (Spalart 1988). In the presented calculations the fringe region has been typically chosen to take up about $5 \%$ of the computational domain on each side.

\subsection{Initial and boundary conditions of the simulations}

Numerical experiments are performed to simulate the linear behaviour of the threedimensional flow resolved in a domain $\left\{-L_{x} \leqslant x \leqslant L_{x}\right\} \times\left\{0 \leqslant y \leqslant L_{y}\right\} \times\{0 \leqslant z \leqslant$ $\left.2 \pi / L_{z}\right\}$. The extended GH model provides the initial conditions for the simulations reported in what follows. Specifically, the initially imposed field has the structure

$$
\begin{aligned}
& \left(\begin{array}{c}
u \\
v \\
w
\end{array}\right)=\frac{1}{\operatorname{Re}}\left(\begin{array}{c}
x \bar{u} \\
\bar{v} \\
\operatorname{Re} \bar{w}
\end{array}\right)
\end{aligned}
$$

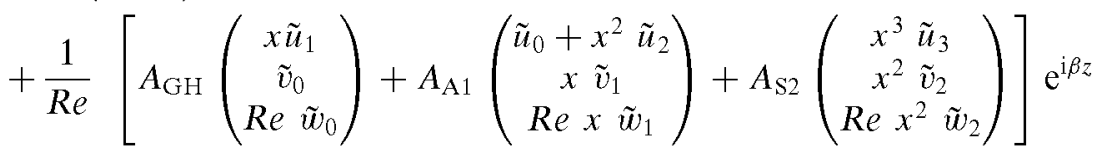

$$
\begin{aligned}
& + \text { c.c. }
\end{aligned}
$$

from which the components of the vorticity vector are calculated. The Reynoldsnumber-dependent scaling of the disturbance eigenfunctions has been introduced for consistency with the basic-flow scaling. This scaling results in an equivalent form of the extended GH model, in which the maximum of the disturbance velocity vector is attained in the wall-normal velocity component. All other qualitative features of the model coefficients are analogous to those discussed in $\$ 5.3$, an increase of mode index being associated with exponential decrease of the magnitude of the corresponding amplitude function, the latter scaled on the respective component of the GH or the A1 mode.

The nonlinear initial boundary value problem is marched in time subject to viscous Dirichlet boundary conditions for the disturbance velocity components at the wall and the far-field and consistently derived Neumann boundary conditions for the disturbance vorticity components. The growth/damping rates are calculated from the DNS signal using an arbitrarily defined measure of modal energy

$$
\varepsilon(\beta, t)=\frac{1}{2 L^{\prime}} \int_{0}^{L_{y}} \mathrm{~d} y \int_{-L^{\prime}}^{L^{\prime}} \frac{1}{2} \boldsymbol{u} \cdot \boldsymbol{u} \mathrm{d} x,
$$

where $\boldsymbol{u}$ is the disturbance velocity vector. The growth rate of a specific eigenmode $\beta$ is calculated from the first time derivative of $\varepsilon(\beta, t)$ using second-order-accurate backward finite-difference formulae. The arbitrariness in the definition of $\varepsilon$ stems from the ambiguity in the choice of the domain $x<\left|L^{\prime}\right|$ in which the influence of the fringe region on the simulation results can be considered negligible. 

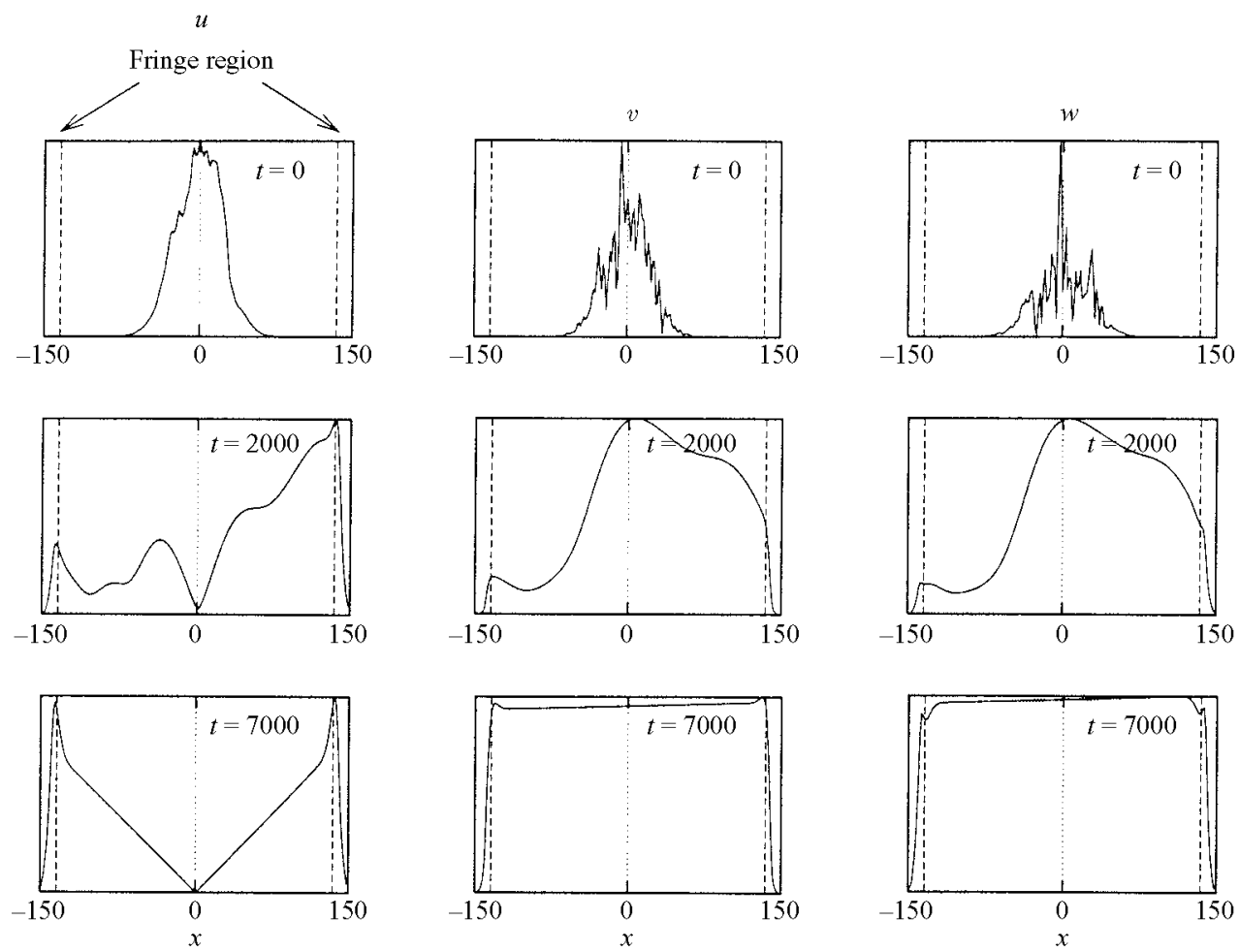

Figure 10. Spalart's test at $R e=600, \beta=0.2845$. Plotted are the maximum r.m.s. values of $\hat{u}, \hat{v}$ and $\hat{w}$ showing the emergence of the GH mode from noise in the simulation (Obrist 2000).

\subsection{Validation and linear runs}

Our first concern is recovery of the linear GH mode, which has been established both experimentally (Poll 1979) and numerically (Hall et al. 1984; Spalart 1988) as being the most relevant linear instability of the attachment-line region. To this end we perform the analogue of the simulation of Spalart (1988), initializing all quantities with white noise superimposed upon the basic state while setting $A_{\mathrm{GH}}=A_{\mathrm{A} 1}=A_{\mathrm{S} 2}=0$ in (7.5), and integrating the equations of motion in time at the linearly unstable parameters $R e=600, \beta=0.2845$. We have taken $L_{y}=150$ and used $(N x, N y)=(192,128)$ collocation points to resolve the chordwise and wall-normal directions respectively. A variable number of collocation points (more than 16 for reasons related to code performance) have been used to resolve the spanwise direction. The higher resolution of the wall-normal direction compared with Spalart (1988) was found to be unnecessary for the purposes of the present validation. The result may be found in graphical form in figure 10 where the GH linear eigenmode can be seen to emerge from noise as time progresses.

We then turn to quantitative comparisons between the results of the temporal ordinary-differential-equation eigenvalue problems (4.40)-(4.41) and (4.42)-(4.43) and the DNS results, with a twofold objective. First, we wish to examine the quality of the recovery of linear results by the DNS, thereby assessing the minimum resolution requirements for nonlinear runs performed subsequently; these results are presented elsewhere (Theofilis \& Obrist 2003). Second, the preservation by our simulation code 
(a)
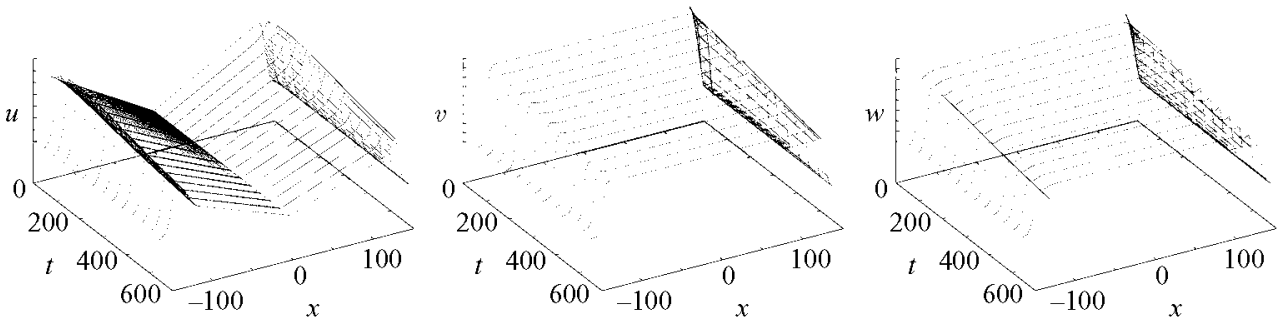

(b)
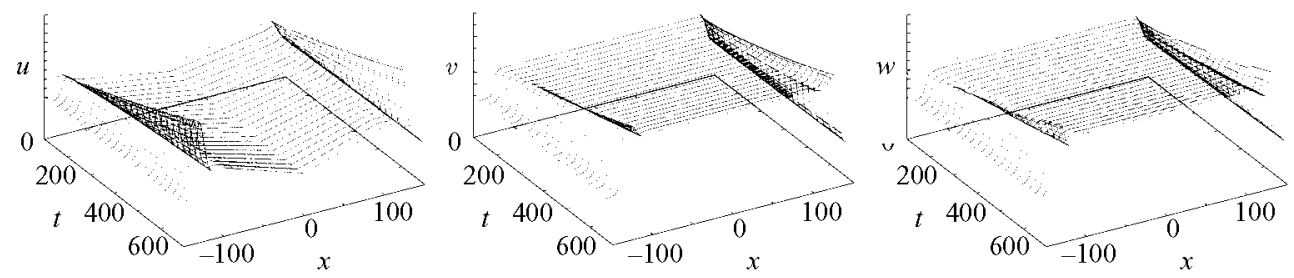

(c)
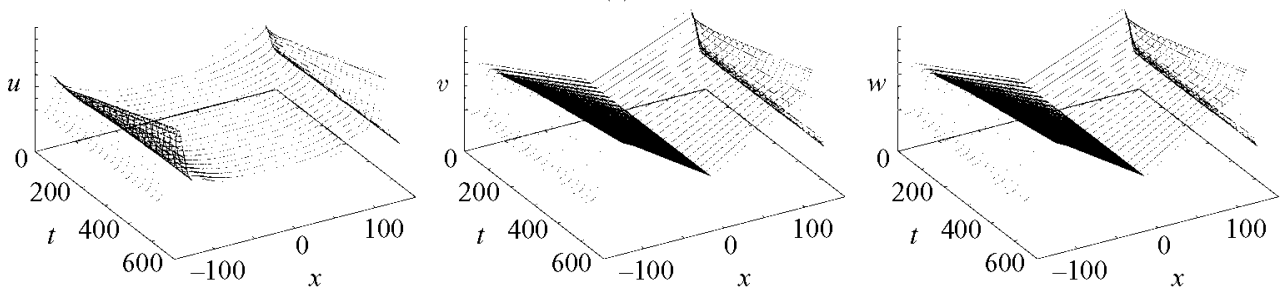

FIgURE 11. The spatial structure of linear eigenmodes obtained by the DNS, in which the imposed symmetry is preserved. (a) subcritical GH run $(R e=550) ;(b)$ supercritical GH run $(R e=800) ;(c)$ supercritical A1 run $(R e=800)$.

of the symmetries of the eigenmodes in the attachment-line region is an additional test to which the code is subjected. Figure 11 shows the r.m.s. over $z$ of the Fourier coefficients $(u, v, w)^{\mathrm{T}}$ of the disturbance eigenfunctions $(\tilde{u}, \tilde{v}, \tilde{w})^{\mathrm{T}}$, taken at their respective maxima along $y$, as functions of $x$ and $t$. The results of three simulations using the linear version of the DNS code, $\beta=0.25$ and a resolution of $(N x, N y, N z)=$ $(192,96,16)$ collocation points are presented. The first two simulations are initialized on the $\mathrm{GH}$ eigenmode by setting $A_{\mathrm{GH}} \neq 0, A_{\mathrm{A} 1}=A_{\mathrm{S} 2}=0$ in (7.5); two Reynolds number values are monitored, $R e=550$ and 800 , respectively corresponding to linearly stable and unstable conditions. An unstable A1 eigenmode at $R e=800$ is used as initial condition for the third simulation using $A_{\mathrm{A} 1} \neq 0$ and $A_{\mathrm{GH}}=A_{\mathrm{S} 2}=0$ in (7.5). Worthy of mention here are the following points.

From a numerical point of view one notices that the fringe treatment (7.3)-(7.4) performs as designed, permitting all three velocity components to develop in the centre part of the calculation domain and smoothly reducing them to zero at the boundaries. From a physical point of view, the imposed linear dependence of the chordwise and independence of the wall-normal and spanwise disturbance velocity components of the GH mode on $x$ are preserved by the time-accurate algorithm; analogously, the quadratic/linear/linear structure of the respective disturbance velocity components 
(a)
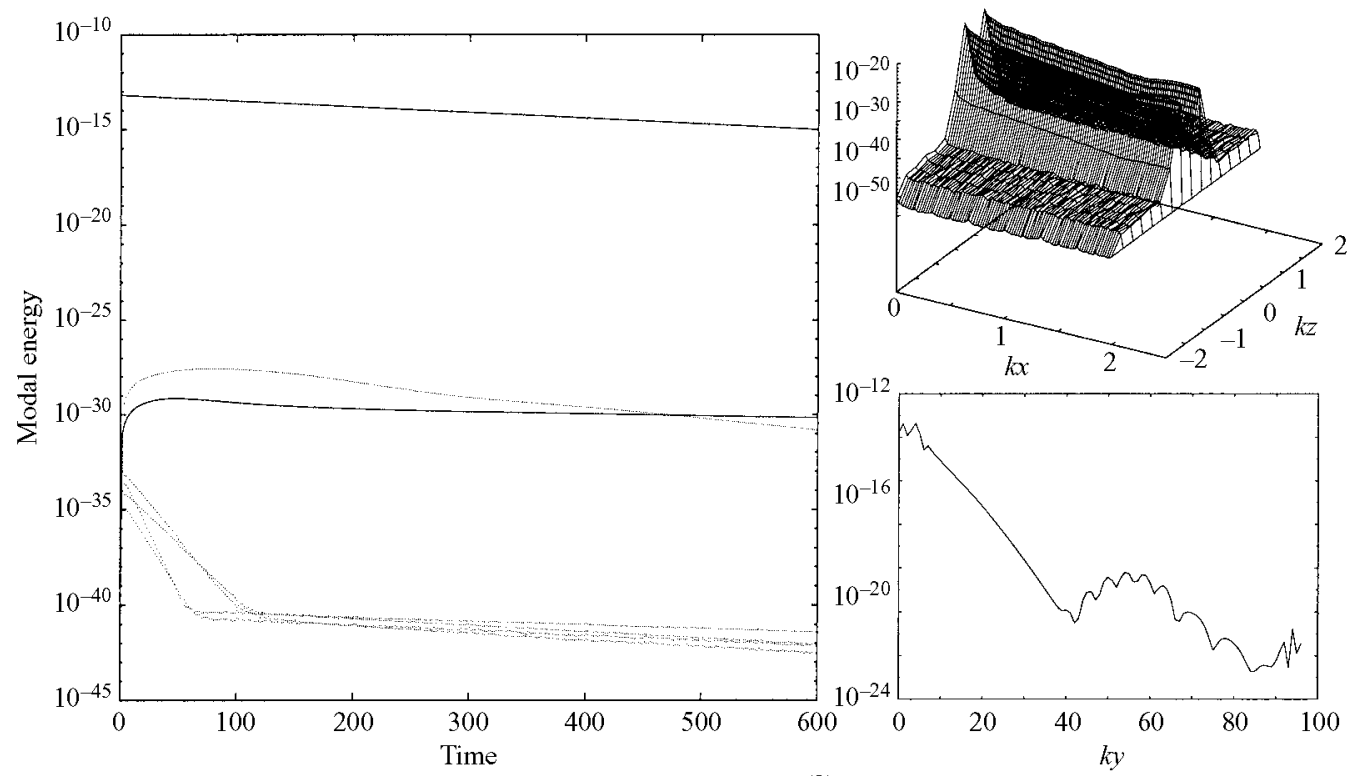

(b)
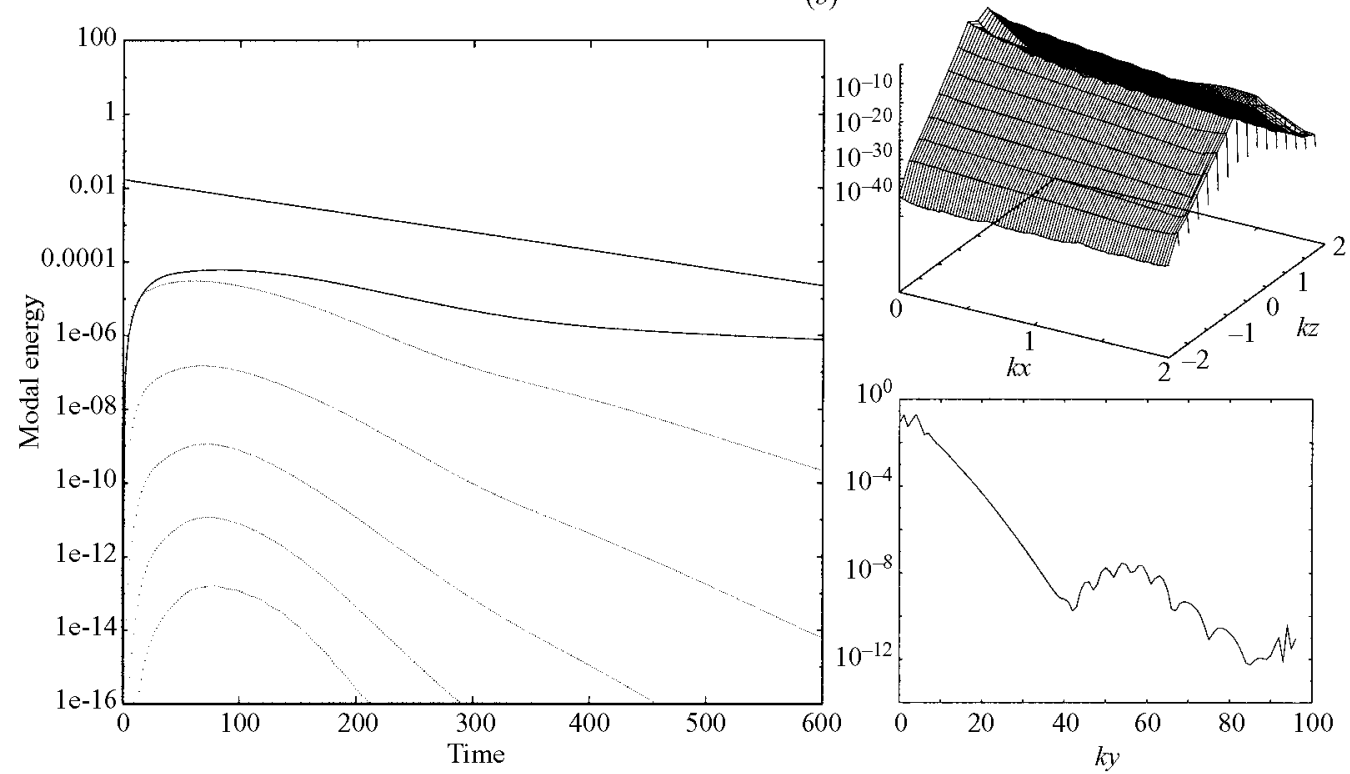

FIGURE 12. (a) Subcritically stable flow. Simulation initialized using a single GH eigenmode at initial amplitude $A=10^{-8}$ and $R e=400$. Solid lines denote the GH mode and the nonlinearly generated mean flow deformation (MFD). All nonlinearly generated modes are stable at these parameters. Also shown is the wavenumber spectrum in the three spatial directions at $t=600$. (b) Subcritically stable flow. Simulation initialized using the GH/A1/S2 eigenmodes. The wavenumber spectra are shown in both cases and all three spatial directions at $t=600$.

of the A1 mode, postulated in (4.2), can be seen in the results of figure 11, including a structure of the chordwise component of the disturbance velocity which is dominated by $\tilde{u}_{0}$ as $x \rightarrow 0$ and by $x^{2} \tilde{u}_{2}$ at large $x$ values. The damping rate of the GH 
mode obtained in the subcritical simulation is $\omega_{i, \mathrm{GH}, \text { sub,DNS }}=-8.43 \times 10^{-4}$ while the growth rates of the supercritical simulations are $\omega_{i, \mathrm{GH}, \text { sup,DNS }}=1.42 \times 10^{-3}$ and $\omega_{i, \mathrm{~A} 1 \text {,sup,DNS }}=9.77 \times 10^{-4}$ for the GH and A1 modes, respectively. The first two quantities are in excellent agreement with the results of the DNS of Theofilis (1998b) in which the chordwise dependence of the equations was formally neglected. The respective predictions of (4.21)-(4.24) and (4.35)-(4.38) are $\omega_{i, \mathrm{GH}, \text { sub,LST }}=-8.41 \times$ $10^{-4}, \omega_{i, \mathrm{GH} \text {,sup,LST }}=1.43 \times 10^{-3}$ and $\omega_{i, \mathrm{~A} 1 \text {,sup,LST }}=9.76 \times 10^{-4}$, which are also in satisfactory agreement with the present DNS results.

Next we turn our attention to nonlinear runs, initialized at linearly low levels of the amplitude of the eigenmode imposed as initial condition in the simulation. The resolution is kept the same as that for the linear runs but the Reynolds number is chosen to be linearly subcritical ( $R e=400, \beta=0.25$ ). The initial condition is formed by a single GH mode at initial amplitude $A_{G H}=10^{-8}$. The time-history of the energy of this mode, as well as those generated nonlinearly, is shown in figure 12(a). In the same figure the energy distribution over the Fourier and Chebyshev modes used in the simulation is presented as function of the wavenumbers $k x, k y$ and $k z$ in the three spatial directions $x, y$ and $z$, respectively. Exponential decay of the spectrum can be seen in all three directions, indicating the adequacy of the resolution of the result presented. Note also that the initial condition is an exact solution of the equations of motion, which is manifested in the absence of transient behaviour in the early stages of linear decay of the GH mode. This behaviour of the code has also been verified in the case of higher amplitudes of the initial condition in (7.5); as an example, amplitudes $A_{\mathrm{GH}}=A_{\mathrm{A} 1}=A_{\mathrm{S} 2}$ are imposed, such that non-negligible nonlinearity is generated while the flow remains stable at all times. The modal energy result is also presented in figure 12 alongside the resolution of the simulation at $t=600$. Here too, linear decay of the initial condition may be seen, while the nonlinearly generated modes are substantially stronger than the result of the previous simulation, in accordance with the increase of the amplitude of the initial condition. The resolution in the wall-normal and chordwise directions exhibit an analogous qualitative behaviour to the linear result, while the increase in the number of modes necessary to describe the spanwise direction is evident, as is the exponential decay in the energy distribution towards the higher modes in all three spatial directions. In other words this class of simulations is also well-resolved. These DNS results further substantiate the threedimensional structure of the eigenmodes as predicted by the extended GH model. The adequate performance of the code is also demonstrated and gives confidence in applying the code to study the nonlinear evolution of disturbances based on the extended GH model. Such nonlinear results will be presented elsewhere (Theofilis \& Obrist 2003).

\section{Discussion}

The new insight into the instability of the incompressible infinite swept attachmentline boundary layer gained by the results presented herein can be summarized as follows. Numerical solutions of the two-dimensional partial-derivative eigenvalue problem which make no use of the symmetries of the swept Hiemenz basic state and which employ a large integration domain in the resolved flow-acceleration and the wall-normal spatial directions have fully confirmed the eigenvalue problem results of Lin \& Malik (1996a). To the extent that statements may be made on the basis of numerical evidence, these modes are found to be the only linear disturbances which 
have a harmonic behaviour in the direction along the attachment line and are relevant to the instability of the attachment-line region in three spatial dimensions. The spatial structure of all two-dimensional amplitude functions in the chordwise direction has been identified herein for the first time, giving rise to the subsequent modelling work.

An extension of the Görtler-Hämmerlin (1955) model, which accounts for symmetry and antisymmetry of the linear disturbances, has been presented. It permits recasting the partial-derivative eigenvalue problem as a sequence of ordinary-differentialequation-based eigenvalue problems (in the wall-normal direction, $y$ ) governing the two respective families of symmetric and antisymmetric instabilities; a polynomial structure of the three-dimensional disturbances in the chordwise direction, $x$, is revealed. In this spatial direction three-dimensional disturbances are expanded in series, the coefficients of which have been shown to decay exponentially with increasing truncation parameter. This, on the one hand results in finite predictions being given by the extended GH model at large $x$ values and on the other hand points to the resolution of an apparent dispute in the literature regarding the correct form of pressure-disturbance eigenmodes.

Results of the proposed one-dimensional extended GH model, in either its temporal or spatial form, are in excellent agreement with those of past investigations based on solutions of the temporal or spatial two-dimensional eigenvalue problem or spatial DNS, with the added benefit of an analytically known dependence of the twodimensional amplitude functions on the chordwise direction. Asymptotic analysis based on a multiple-scales expansion in terms of an inverse-Reynolds-number small parameter has been employed independently to verify the results of the extended GH model; this methodology too demonstrates that the polynomial (in $x$ ) structure of the two-dimensional amplitude functions is analysable and that two-dimensional (in $x$ and $y$ ) eigenvalue problem results may be obtained by solution of a sequence of one-dimensional (in $y$ ) eigenvalue problems. In the light of these results, either of the other two alternative approaches currently in use to obtain three-dimensional linear instability characteristics in the swept Hiemenz boundary layer, namely solution of the temporal or spatial partial derivative eigenvalue problem or performance of a spatial DNS, becomes superfluous.

Turning to comparisons with experiment, a factor potentially leading to confusing the higher polynomial modes with the predominant GH instability is that the frequencies of all discrete modes lie very close to each other and are placed well within the error bar of available experimental data (Lin \& Malik 1996a; Theofilis $1998 \mathrm{~b}$ ). Interestingly, all two-dimensional amplitude functions of three-dimensional eigenmodes in the attachment-line boundary layer have been shown here to possess a unique (scaled) dependence on the wall-normal coordinate, that of the well-known GH mode. In most stability experiments one-dimensional profiles are (traditionally) expected and consequently measured. Both these results may explain why the additional modes were never reported experimentally as individual solutions in their own right in experiments which have exclusively focused on frequency measurements and are yet to provide even the one-dimensional (in $y$ ) structure of a linear eigenmode. Two criteria for the experimental isolation of the three-dimensional modes may be used. The first exists in the results of earlier work, namely that, unlike the result on frequency, each mode possesses a growth rate which is well separated from that of the other modes. Modes may thus be isolated by appropriate selection of the Reynolds number and frequency parameters; the spatial form of the extended GH model solved herein may be utilized in order to obtain the parameter values sought in 
an efficient manner. The second criterion follows from the present analysis. Alongside measurements in the wall-normal direction, probes must be placed along the chord. With the spatial structure of eigenvectors usually recovered experimentally by curve fits through measurement points, the number of stations at which measurements must be made is a function of the mode sought. Two hot wires are only sufficient for the GH disturbance which grows linearly in $x$; the spatial structure of all other modes requires a number of probes which follows from the $x$-dependence of the mode monitored, as shown in model (4.1) - (4.2). Alternatively, modern experimental techniques capable of providing information on the entire $(O, x, y)$-plane might become interesting in this flow. The theoretical results provided herein are expected to provide a focus for necessary new experimental efforts in this respect.

Finally, from a theoretical point of view, modes with identical $y$-dependence and simple prescribed analytical $x$-dependence which have approximately equal frequencies may be utilized to build analytical models in the framework of nonlinear calculations. The results of one such effort, in which three-dimensional direct numerical simulations were performed utilizing the extended GH model in order to construct physically plausible initial conditions, are presented in an accompanying paper (Theofilis \& Obrist 2003).

This work was performed while the first author was in receipt of an Alexander von Humboldt Research Fellowship.

\section{Appendix. Implementation of the companion matrix approach for the spatial linear stability eigenvalue problem}

The system (4.40)-(4.41) may be recast as a spatial eigenvalue problem by the introduction of the vector of unknowns $X=\left(\tilde{u}_{2 M-1}, \beta \tilde{u}_{2 M-1}, \tilde{v}_{2 M-2}, \beta \tilde{v}_{2 M-2}, \beta^{2} \tilde{v}_{2 M-2}\right.$, $\left.\beta^{3} \tilde{v}_{2 M-2}\right)^{T}$. The matrices $\boldsymbol{A}$ and $\boldsymbol{B}$ of the resulting spatial eigenproblem $\boldsymbol{A} X=$ $\beta \boldsymbol{B} X$ have the following non-zero submatrices, resulting from collocation of the equations:

$$
\begin{aligned}
& A_{11}=\mathscr{D}^{2}-\bar{V} \mathscr{D}-2 M \bar{U}+\mathrm{i} \Omega R e, \\
& A_{13}=-\bar{U}^{\prime}, \\
& A_{31}=2(2 M-1)\left(\bar{U} \mathscr{D}+\bar{U}^{\prime}\right), \\
& A_{33}=\mathscr{D}^{4}-\bar{V} \mathscr{D}^{3}-\left[2(M-1) \bar{U}+\bar{V}^{\prime}-\mathrm{i} \Omega \operatorname{Re}\right] \mathscr{D}^{2}+\bar{U}^{\prime} \mathscr{D}+(2 M-1) \bar{U}^{\prime \prime}, \\
& B_{11}=\mathrm{i} \operatorname{Re} \bar{W} \\
& B_{12}=I, \\
& B_{33}=\mathrm{i} \operatorname{Re} \bar{W} \mathscr{D}^{2}-\mathrm{i} \operatorname{Re} \bar{W}^{\prime \prime}, \\
& B_{34}=2 \mathscr{D}^{2}-\bar{V} \mathscr{D}-\left[2(M-1) \bar{U}+\bar{V}^{\prime}-\mathrm{i} \Omega \operatorname{Re}\right], \\
& B_{35}=-\mathrm{i} \operatorname{Re} \bar{W}, \\
& B_{36}=-I,
\end{aligned}
$$

while the auxiliary matrices which have to be introduced are

$$
A_{22}=A_{44}=A_{55}=A_{66}=B_{21}=B_{43}=B_{54}=B_{65}=I .
$$

The boundary conditions $\tilde{u}_{2 M-1}=\tilde{v}_{2 M-2}=0$ and $\mathscr{D} \tilde{v}_{2 M-2}=0$ at both ends of the integration domain complete the solution algorithm for the symmetric modes. In an analogous manner, antisymmetric disturbances satisfying (4.42)-(4.43) may be 
obtained by introducing the vector of unknowns $X=\left(\tilde{u}_{2 M}, \beta \tilde{u}_{2 M}, \tilde{v}_{2 M-1}, \beta \tilde{v}_{2 M-1}\right.$, $\left.\beta^{2} \tilde{v}_{2 M-1}, \beta^{3} \tilde{v}_{2 M-1}\right)^{T}$, collocating the submatrices

$$
\begin{aligned}
& A_{11}=\mathscr{D}^{2}-\bar{V} \mathscr{D}-(2 M+1) \bar{U}+\mathrm{i} \Omega R e, \\
& A_{13}=-\bar{U}^{\prime}, \\
& A_{31}=4 M\left(\bar{U} \mathscr{D}+\bar{U}^{\prime}\right), \\
& A_{33}=\mathscr{D}^{4}-\bar{V} \mathscr{D}^{3}-\left[(2 M-1) \bar{U}+\bar{V}^{\prime}-\mathrm{i} \Omega \operatorname{Re}\right] \mathscr{D}^{2}+\bar{U}^{\prime} \mathscr{D}+2 M \bar{U}^{\prime \prime}, \\
& B_{11}=\mathrm{i} \operatorname{Re} \bar{W} \\
& B_{12}=I, \\
& B_{33}=\mathrm{i} e \bar{W} \mathscr{D}^{2}-\mathrm{i} \operatorname{Re}^{\prime \prime}, \\
& B_{34}=2 \mathscr{D}^{2}-\bar{V} \mathscr{D}-\left[(2 M-1) \bar{U}+\bar{V}^{\prime}-\mathrm{i} \Omega \operatorname{Re}\right], \\
& B_{35}=-\mathrm{i} \operatorname{Re} \bar{W}, \\
& B_{36}=-I,
\end{aligned}
$$

and

$$
A_{22}=A_{44}=A_{55}=A_{66}=B_{21}=B_{43}=B_{54}=B_{65}=I,
$$

and solving the resulting eigenproblem subject to the boundary conditions $\tilde{u}_{2 M}=$ $\tilde{v}_{2 M-1}=0$ and $\mathscr{D} \tilde{v}_{2 M-1}=0$ at the wall, $y=0$, and the location where the $y$-integration domain is truncated, $y=y_{\infty}$.

\section{REFERENCES}

Arnal, D., Coustols, R. \& Juillen, J. 1984 Etude expérimentale et théorique de la transition sur une ailc en fléche infinie. La Recherche Aérospatiale 4, 275-290.

Balakumar, P. 1998 Finite amplitude stability of attachment-line boundary layers. AIAA Paper 98-0338.

Bertolotti, F. P. 2000 On the connection between cross-flow vortices and attachment-line instabilities. In Proc. IUTAM Laminar-Turbulent Symposium V, Sedona, AZ, USA (ed. W. Saric \& H. Fasel), pp. 625-630.

BoYD, J. 1989 Chebyshev and Fourier Spectral Methods. Springer.

Bridges, T. \& Morris, P. 1984 Differential eigenvalue problems in which the parameter appears nonlinearly. J. Comput. Phys. 55, 437.

Canuto, C., Hussaini, M. Y., Quarteroni, A. \& Zang, T. A. 1987 Spectral Methods in Fluid Dynamics. Springer.

Dallmann, U. 1980 Zur Stabilität dreidimensionaler Scherströmungen. Kriterien für reibungsfreie Störungsanregung. Tech. Rep. FB 80-05. DLR.

Drazin, P. \& ReID, W. 1981 Hydrodynamic Stability. Cambridge University Press.

Ehrenstenn, U. 1996 On the linear stability of channel flows over riblets. Phys. Fluids 8, 31943196.

Gaster, M. 1967 On the flow along swept leading edges. Aero. Q. 18, 165-184.

GöRTLER, H. 1955 Dreidimensionale Instabilität der ebenen Staupunktströmung gegenüber wirbelartigen Störungen. In 50 Jahre Grenzschichtforschung (ed. H. Görtler \& W. Tollmien), pp. 304-314. Vieweg und Sohn.

Hall, P. \& Malik, M. 1986 On the instability of a three-dimensional attachment-line boundary layer: weakly nonlinear theory and a numerical approach. J. Fluid Mech. 163, 257-282. 
Hall, P., Malik, M. \& Poll, D. 1984 On the stability of an infinite swept attachment-line boundary layer. Proc. R. Soc. Lond. A 395, 229-245.

Hall, P. \& SEDdougui, S. 1990 Wave interactions in a three-dimensional attachment-line boundary layer. J. Fluid Mech. 217, 367-390.

HÄmmeRLIN, G. 1955 Zur Instabilitätstheorie der ebenen Staupunktströmung. In 50 Jahre Grenzschichtforschung (ed. H. Görtler \& W. Tollmien), pp. 315-327. Vieweg und Sohn.

Heeg, R. \& GeurTs, B. 1998 Spatial instabilities of incompressible attachment-line flow using sparse matrix Jacobi-Davidson techniques. Appl. Sci. Res. 59, 315-329.

Hiemenz, K. 1911 Die Grenzschicht an einem in den gleichförmigen Flüssigkeitsstrom eingetauchten geraden Kreiszylinder. Dingl. Polytechn. J. 326, 321-324, thesis, Göttingen.

Jiménez, J., Martel, C., AGüÍ, J. \& ZufiRiA, J. 1990 Direct numerical simulation of transition in the incompressible leading edge boundary layer. Tech. Rep. MF-903. ETSIA.

JosLIN, R. 1995 Direct simulation of evolution and control of three-dimensional instabilities in attachment-line boundary layers. J. Fluid Mech. 291, 369-392.

JosLIN, R. D. 1996 a Simulation of nonlinear instabilities in an attachment-line boundary layer. Fluid Dyn. Res. 18, 81-97.

JosLIN, R. D. $1996 b$ Simulation of three-dimensional symmetric and antisymmetric instabilities in attachment-line boundary layers. AIAA J. 34, 2432-2434.

Josin, R. D. 1997 Direct numerical simulation of evolution and control of linear and nonlinear disturbances in three-dimensional attachment-line boundary layers. Tech. Rep. TP 3623. NASA.

LIN, R.-S. \& MALIK, M. R. $1996 a$ On the stability of attachment-line boundary layers. Part 1. the incompressible swept hiemenz flow. J. Fluid Mech. 311, 239-255.

LN, R.-S. \& MALIK, M. R. $1996 b$ On the stability of attachment-line boundary layers. Part 2. the effect of leading-edge curvature. J. Fluid Mech. 333, 125-137.

Lundbladh, A., Schmid, P., Berlin, S. \& Henningson, D. 1994 Simulation of bypass transition in spatially evolving flow. In $A G A R D C P-551$ (ed. B. Cantwell), pp. 18-1-18-13.

NAYFEH, A. H. 1980 Stability of three-dimensional boundary layers. AIAA. J. 18, 406-416.

OBRIst, D. 2000 On the stability of the swept leading-edge boundary layer. $\mathrm{PhD}$ thesis, University of Washington.

Pfenninger, W. \& Bacon, J. 1969 Amplified laminar boundary layer oscillation and transition at the front attachment line of a $45^{\circ}$ swept flat-nosed wing with and without suction. In Viscous Drag Reduction (ed. C. S. Wells), pp. 85-105. Plenum Press.

Poll, D. 1979 Transition in the infinite swept attachment line boundary layer. Aero. Q. 30, 607629.

Poll, D., Danks, M. \& Yardley, M. 1996 The effects of suction and blowing on stability and transition at a swept attachment line. In Transitional Boundary Layers in Aeronautics (ed. R. Henkes \& J. van Ingen). Elsevier.

RosenHEAD, L. 1963 Laminar Boundary Layers. Oxford University Press.

SAAD, Y. 1980 Variations of arnoldi's method for computing eigenelements of large unsymmetric matrices. Lin. Algebra Appl. 34, 269-295.

SPALART, P. 1988 Direct numerical study of leading-edge contamination. In AGARD CP-438, pp. 5-1-5-13.

SPALART, P. 1989 Direct numerical study of crossflow instability. In IUTAM Laminar-Turbulent Symposium III (ed. D. Arnal \& R. Michel), pp. 621-630.

TheofiLis, V. 1995 Spatial stability of incompressible attachment line flow. Theor. Comput. Fluid Dyn. 7, 159-171.

TheofiLIS, V. 1997 On the verification and extension of the Görtler-Hämmerlin assumption in three-dimensional incompressible swept attachment-line boundary layer flow. Tech. Rep. IB 223-97 A 44. DLR.

Theofilis, V. 1998 a Linear instability in two spatial dimensions. In Proc. European Computational Fluid Dynamics Conf. ECCOMAS '98 (ed. K. D. Papailiou, D. Tsahalis, J. Périaux, C. Hirsch \& M. Pandolfi), Athens, Greece, pp. 547-552. John Wiley \& Sons.

TheofiLIs, V. $1998 b$ On linear and nonlinear instability of the incompressible swept attachment-line boundary layer. J. Fluid Mech. 355, 193-227.

TheofiLis, V. 2003 Advances in global linear instability analysis of nonparallel and three-dimensional flows. Prog. Aero. Sci. 39, 249-315. 
Theofilis, V., Hein, S. \& Dallmann, U. 2000 On the origins of unsteadiness and threedimensionality in a laminar separation bubble. Phil. Trans. R. Soc. Lond. A. 358, 3229-324.

Theofilis, V. \& OBRist, D. 2003 The extention of the Görtler-Hämmerlin model for linear instability in the three-dimensional incompressible swept attachment-line boundary layer. Part 2. Nonlinear mode interactions. In preparation.

VAn Dyke, M. 1975 Perturbation Methods in Fluid Mechanics. Parabolic.

Zhigulev, V. \& Tumin, A. 1987 Occurrence of Turbulence. Nauka, Novosibirsk (in Russian). Translated as NASA TT-20340. 